\title{
Asymptotic bounds for the sizes of constant dimension codes and an improved lower bound
}

\author{
Daniel Heinlein and Sascha Kurz* \\ University of Bayreuth \\ Daniel.Heinlein@uni-bayreuth.de, Sascha.Kurz@uni-bayreuth.de \\ May 10, 2017
}

\begin{abstract}
We study asymptotic lower and upper bounds for the sizes of constant dimension codes with respect to the subspace or injection distance, which is used in random linear network coding. In this context we review known upper bounds and show relations between them. A slightly improved version of the so-called linkage construction is presented which is e.g. used to construct constant dimension codes with subspace distance $d=4$, dimension $k=3$ of the codewords for all field sizes $q$, and sufficiently large dimensions $v$ of the ambient space, that exceed the MRD bound, for codes containing a lifted MRD code, by Etzion and Silberstein.
\end{abstract}

Keywords: constant dimension codes, subspace distance, injection distance, random network coding

\section{Introduction}

Let $V \cong \mathbb{F}_{q}^{v}$ be a $v$-dimensional vector space over the finite field $\mathbb{F}_{q}$ with $q$ elements. By $\left[\begin{array}{l}V \\ k\end{array}\right]$ we denote the set of all $k$-dimensional subspaces in $V$, where $0 \leq k \leq v$, which has size $\left[\begin{array}{l}v \\ k\end{array}\right]_{q}:=\prod_{i=1}^{k} \frac{q^{v-k+i}-1}{q^{i}-1}$. More general, the set $P(V)$ of all subspaces of $V$ forms a metric space with respect to the subspace distance defined by $\mathrm{d}_{\mathrm{s}}(U, W)=$ $\operatorname{dim}(U+W)-\operatorname{dim}(U \cap W)=\operatorname{dim}(U)+\operatorname{dim}(W)-2 \operatorname{dim}(U \cap W)$, see [32], and the injection distance defined by $\mathrm{d}_{\mathbf{i}}(U, W)=\max \{\operatorname{dim}(U), \operatorname{dim}(W)\}-\operatorname{dim}(U \cap W)$, see [40].

\footnotetext{
${ }^{*}$ The work was supported by the ICT COST Action IC1104 and grants KU 2430/3-1, WA 1666/9-1 "Integer Linear Programming Models for Subspace Codes and Finite Geometry" - from the German Research Foundation.
} 
Coding Theory on $P(V)$ is motivated by Kötter and Kschischang [32] via error correcting random network coding, see [4]. In this context it is natural to consider codes $\mathcal{C} \subseteq P(V)$ where each codeword, i.e., each element of $\mathcal{C}$, has the same dimension $k$, called constant dimension code (cdc), since this knowledge can be exploited by decoders. For constant dimension codes we have $\mathrm{d}_{\mathrm{s}}(U, W)=2 \mathrm{~d}_{\mathrm{i}}(U, W)$, so that we will only consider the subspace distance in this paper. By $(v, N, d ; k)_{q}$ we denote a cdc in $V$ with minimum (subspace) distance $d$ and size $N$, where the dimensions of each codeword is $k \in\{0,1, \ldots, v\}$. As usual, a cdc $\mathcal{C}$ has the minimum distance $d$, if $d \leq \mathrm{d}_{\mathrm{s}}(U, W)$ for all $U \neq W \in \mathcal{C}$ and equality is attained at least once. If $\# \mathcal{C}=1$, we set the minimum distance to $\infty$. The corresponding maximum size is denoted by $A_{q}(v, d ; k)$, where we allow the minimum distance to be larger than $d$. In [32] the authors provided lower and upper bounds for $A_{q}(v, d ; k)$ which are less than a factor of 4 apart. For increasing field size $q$ this factor tends to 1 . Here, we tighten the corresponding analysis and end up in a factor of less than 2 for the binary field $q=2$ and a strictly better factor for larger values of $q$. With respect to lower bounds, we slightly generalize the so-called linkage construction by Gluesing-Luerssen, Troha / Morrison [22, 21] and Silberstein, (Horlemann-)Trautmann [38. This improvement then gives the best known lower bounds for $A_{q}(v, d ; k)$ for many parameters, cf. the online tables http://subspacecodes.uni-bayreuth.de associated with [23]. For codes containing a lifted maximum rank distance (LMRD) code as a subcode an upper bound on the size has been presented in [16] for some infinite series of parameters. Codes larger than this MRD bound are very rare. Based on the improved linkage construction we give an infinite series of such examples.

In this context we mention the following asymptotic result based on the non-constructive probabilistic method. If the subspace distance $d$ and the dimension $k$ of the codewords is fixed, then the ratio of the lower and upper bound tends to 1 as the dimension $v$ of the ambient space approaches infinity, see [18, Theorem 4.1], which is implied by a more general result of Frankl and Rödl on hypergraphs. The same result, with an explicit error term, was also obtained in [8, Theorem 1]. If $d$ and $v-k$ is fixed we have the same result due to the orthogonal code. If the parameter $k$ can vary with the dimension $v$, then our asymptotic analysis implies there is still a gap of almost 2 between the lower and the upper bound of the code sizes for $d=4$ and $k=\lfloor v / 2\rfloor$, which is the worst case.

The remaining part of the paper is organized as follows. In Section 2 we collect the basic facts and definitions for constant dimension codes. Upper bounds on the achievable codes sizes are reviewed in Section 3. Here, we partially extend the current knowledge on the relation between these bounds. While most of them are known around 2008 there are some recent improvements for the subclass of partial spreads, where $d=2 k$, which we summarize in Subsection 3.1. In Section 4 we present the mentioned improvement of the linkage construction. Asymptotic bounds for the ratio between lower and upper bounds for code sizes are studied in Section 5. We continue with the upper bound for constant dimension codes containing a lifted MRD code in Section 6, including some numerical results, before we draw a short conclusion in Section 7 . 


\section{Preliminaries}

For the remainder of the paper we set $V \cong \mathbb{F}_{q}^{v}$, where $q$ is a prime power. By $v$ we denote the dimension of $V$. Using the language of projective geometry, we will call the 1-dimensional subspaces of $\mathbb{F}_{q}^{v}$ points and the 2-dimensional subspaces lines. First, we observe that the $q$-binomial coefficient $\left[\begin{array}{l}v \\ k\end{array}\right]_{q}$ indeed gives the cardinality of $\left[\begin{array}{l}V \\ k\end{array}\right]$. To this end, we associate with a subspace $U \in\left[\begin{array}{c}V \\ k\end{array}\right]$ a unique $k \times v$ matrix $X_{U}$ in row reduced echelon form (rref) having the property that $\left\langle X_{U}\right\rangle=U$ and denote the corresponding bijection

$$
\left[\begin{array}{c}
\mathbb{F}_{q}^{v} \\
k
\end{array}\right] \rightarrow\left\{X_{U} \in \mathbb{F}_{q}^{k \times v} \mid \operatorname{rk}\left(X_{U}\right)=k, X_{U} \text { is in rref }\right\}
$$

by $\tau$. An example is given by $X_{U}=\left(\begin{array}{lll}1 & 0 & 0 \\ 0 & 1 & 1\end{array}\right) \in \mathbb{F}_{2}^{2 \times 3}$, where $U=\tau^{-1}\left(X_{U}\right) \in\left[\begin{array}{c}\mathbb{F}_{2}^{3} \\ 2\end{array}\right]$ is a line that contains the three points $(1,0,0),(1,1,1)$, and $(0,1,1)$. Counting those matrices gives

$$
\#\left[\begin{array}{c}
V \\
k
\end{array}\right]=\prod_{i=0}^{k-1} \frac{q^{v}-q^{i}}{q^{k}-q^{i}}=\prod_{i=1}^{k} \frac{q^{v-k+i}-1}{q^{i}-1}=\left[\begin{array}{c}
v \\
k
\end{array}\right]_{q}
$$

for all integers $0 \leq k \leq v$. Especially, we have $\left[\begin{array}{l}v \\ v\end{array}\right]_{q}=\left[\begin{array}{l}v \\ 0\end{array}\right]_{q}=1$. Given a non-degenerate bilinear form, we denote by $U^{\perp}$ the orthogonal subspace of a subspace $U$, which then has dimension $v-\operatorname{dim}(U)$. Then, we have $\mathrm{d}_{\mathrm{s}}(U, W)=\mathrm{d}_{\mathrm{s}}\left(U^{\perp}, W^{\perp}\right)$, so that $\left[\begin{array}{c}v \\ k\end{array}\right]_{q}=\left[\begin{array}{c}v \\ v-k\end{array}\right]_{q}$. The recurrence relation for the usual binomial coefficients generalize to $\left[\begin{array}{l}v \\ k\end{array}\right]_{q}=q^{k}\left[\begin{array}{c}v-1 \\ k\end{array}\right]_{q}+$ $\left[\begin{array}{c}v-1 \\ k-1\end{array}\right]_{q}$. In order to remove the restriction $0 \leq k \leq v$, we set $\left[\begin{array}{l}a \\ b\end{array}\right]_{q}=0$ for $a \in \mathbb{N}_{\geq 0}$ and $b \in \mathbb{Z}$, whenever $b<0$ or $a<b$. This extension goes in line with the interpretation of the number of $b$-dimensional subspaces of $\mathbb{F}_{q}^{a}$ and respects the orthogonality relation. In order to write $\sum_{j=0}^{v-1} q^{j}=\left[\begin{array}{l}v \\ 1\end{array}\right]_{q}$ for positive integers $q$ in later formulas, we apply the definition of $\left[\begin{array}{l}v \\ k\end{array}\right]_{q}$ also in cases where $q$ is not a prime power and $\operatorname{set}\left[\begin{array}{l}v \\ k\end{array}\right]_{1}=\left(\begin{array}{l}v \\ k\end{array}\right)$ for $q=1$.

Using the bijection $\tau$ we can express the subspace distance between two $k$-dimensional subspaces $U, W \in\left[\begin{array}{c}V \\ k\end{array}\right]$ via the rank of a matrix:

$$
\mathrm{d}_{\mathrm{s}}(U, W)=2 \operatorname{dim}(U+W)-\operatorname{dim}(U)-\operatorname{dim}(W)=2\left(\operatorname{rk}\left(\begin{array}{c}
\tau(U) \\
\tau(W)
\end{array}\right)-k\right) .
$$

Using $\left[\begin{array}{l}V \\ k\end{array}\right]$ as vertex set, we obtain the so-called Grassmann graph, where two vertices are adjacent iff the corresponding subspaces intersect in a space of dimension $k-1$. It is well-known that the Grassmann graph is distance regular. The injection distance $\mathrm{d}_{\mathrm{i}}(U, W)$ corresponds to the graph distance in the Grassmann graph. Considered as an association scheme one speaks of the $q$-Johnson scheme.

If $\mathcal{C} \subseteq\left[\begin{array}{c}V \\ k\end{array}\right]$ is a cdc with minimum subspace distance $d$, we speak of a $(v, \# \mathcal{C}, d ; k)$ constant dimension code. In the special case of $d=2 k$ one speaks of so-called partial spreads, i.e., collections of $k$-dimensional subspaces with pairwise trivial intersection.

Besides the injection and the subspace distance we will also consider the Hamming distance $\mathrm{d}_{\mathrm{h}}(u, w)=\#\left\{i \mid u_{i} \neq w_{i}\right\}$, for two vectors $u, w \in \mathbb{F}_{2}^{v}$, and the rank distance $\mathrm{d}_{\mathrm{r}}(U, W)=\operatorname{rk}(U-W)$, for two matrices $U, W \in \mathbb{F}_{q}^{m \times n}$. The latter is indeed a metric, as observed in [20]. A subset $\mathcal{C} \subseteq \mathbb{F}_{q}^{m \times n}$ is called a rank metric code. If the minimum 
rank-distance of $\mathcal{C}$ is given by $d_{r}$, we will also speak of an $\left(m \times n, \# \mathcal{C}, d_{r}\right)_{q}$ rank metric code in order to specify its parameters. A rank metric code $\mathcal{C} \subseteq \mathbb{F}_{q}^{m \times n}$ is called linear if $\mathcal{C}$ forms a subspace of $\mathbb{F}_{q}^{m \times n}$, which implies that $\# \mathcal{C}$ has to be a power of the field size $q$.

Theorem 1. (see [20]) Let $m, n \geq d$ be positive integers, $q$ a prime power, and $\mathcal{C} \subseteq \mathbb{F}_{q}^{m \times n}$ be a rank metric code with minimum rank distance $d$. Then, $\# \mathcal{C} \leq q^{\max \{n, m\} \cdot(\min \{n, m\}-d+1)}$.

Codes attaining this upper bound are called maximum rank distance (MRD) codes. They exist for all (suitable) choices of parameters, which remains true if we restrict to linear rank metric codes, see [20]. If $m<d$ or $n<d$, then only $\# \mathcal{C}=1$ is possible, which can be achieved by a zero matrix and may be summarized to the single upper bound $\# \mathcal{C} \leq\left\lceil q^{\max \{n, m\} \cdot(\min \{n, m\}-d+1)}\right\rceil$. Using an $m \times m$ identity matrix as a prefix one obtains the so-called lifted MRD codes.

Theorem 2. [39, Proposition 4] For positive integers $k, d, v$ with $k \leq v, d \leq 2 \min \{k, v-$ $k\}$, and $d$ even, the size of a lifted MRD code in $\left[\begin{array}{l}V \\ k\end{array}\right]$ with subspace distance $d$ is given by

$$
M(q, k, v, d):=q^{\max \{k, v-k\} \cdot(\min \{k, v-k\}-d / 2+1)} .
$$

If $d>2 \min \{k, v-k\}$, then we have $M(q, k, v, d):=1$.

The Hamming distance can be used to lower bound the subspace distance between two codewords (of the same dimension). To this end let $p:\left\{M \in \mathbb{F}_{q}^{k \times v} \mid \operatorname{rk}(M)=\right.$ $k, \mathrm{M}$ is in rref $\} \rightarrow\left\{x \in \mathbb{F}_{2}^{v} \mid \sum_{i=1}^{v} x_{i}=k\right\}$ denote the pivot positions of the matrix in rref. For our example $X_{U}$ we we have $p\left(X_{U}\right)=(1,1,0)$. Slightly abusing notation we also write $p(U)$ for subspaces $U \in\left[\begin{array}{l}V \\ k\end{array}\right]$ instead of $p(\tau(U))$.

Lemma 1. [15, Lemma 2] For two subspaces $U, W \leq \mathbb{F}_{q}^{v}$, we have $\mathrm{d}_{\mathrm{s}}(U, W) \geq \mathrm{d}_{\mathrm{h}}(p(U), p(W))$.

\section{Upper Bounds}

In this section we review and compare known upper bounds for the sizes of constant dimension codes. Here we assume that $v, d$, and $k$ are integers with $2 \leq k \leq v-2$, $4 \leq d \leq 2 \min \{k, v-k\}$, and $d$ even in all subsequent results. The bound $0 \leq k \leq v$ just ensures that $\left[\begin{array}{c}V \\ k\end{array}\right]$ is non-empty. Note that $\mathrm{d}_{\mathrm{s}}(U, W) \leq 2 \min \{k, v-k\}$ and $\mathrm{d}_{\mathrm{s}}(U, W)$ is even for all $U, W \in\left[\begin{array}{c}V \\ k\end{array}\right]$. Restricting to the set case, we trivially have $A_{q}(v, d ; k)=$ $\#\left[\begin{array}{l}V \\ k\end{array}\right]=\left[\begin{array}{l}v \\ k\end{array}\right]_{q}$ for $d \leq 2$ or $k \leq 1$, so that we assume $k \geq 2$ and $d \geq 4$, which then implies $k \leq v-2$ and $v \geq 4$. We remark that some of the latter bounds are also valid for parameters outside the ranges of non-trivial parameters considered by us. Since the maximum size of a code with certain parameters is always an integer and some of the latter upper bounds can produce non-integer values, we may always round them down. To ease the notation we will commonly omit the final rounding step.

The list of known bounds has not changed much since [29], see also [17]. Comparisons of those bounds are scattered among the literature and partially hidden in comments, see e.g. [6]. Additionally some results turn out to be wrong or need a reinterpretation at the very least. 
Counting $k$-dimensional subspaces having a large intersection with a fixed $m$-dimensional subspace gives:

Lemma 2. For integers $0 \leq t \leq k \leq v$ and $k-t \leq m \leq v$ we have

$$
\#\left\{U \in\left[\begin{array}{l}
V \\
k
\end{array}\right] \mid \operatorname{dim}(U \cap W) \geq k-t\right\}=\sum_{i=0}^{t} q^{(m+i-k) i}\left[\begin{array}{c}
m \\
k-i
\end{array}\right]_{q}\left[\begin{array}{c}
v-m \\
i
\end{array}\right]_{q},
$$

where $W \leq V$ and $\operatorname{dim}(W)=m$.

Proof. Let us denote $\operatorname{dim}(U \cap W)$ by $k-i$, where $\max \{0, k-m\} \leq i \leq \min \{t, v-m\}$. With this, the number of choices for $U$ is given by

$$
\begin{aligned}
& \frac{\left(q^{m}-q^{0}\right) \cdot\left(q^{m}-q^{1}\right) \cdots\left(q^{m}-q^{k-i-1}\right) \cdot\left(q^{v}-q^{m+1}\right) \cdots\left(q^{v}-q^{m+i-1}\right)}{\left(q^{k}-q^{0}\right) \cdot\left(q^{k}-q^{1}\right) \cdots\left(q^{k}-q^{k-1}\right)} \\
= & {\left[\begin{array}{c}
m \\
k-i
\end{array}\right]_{q} \cdot \frac{\left(q^{m}\right)^{i}}{\left(q^{k-i}\right)^{i}} \cdot\left[\begin{array}{c}
v-m \\
i
\end{array}\right]_{q}=q^{(m+i-k) i}\left[\begin{array}{c}
m \\
k-i
\end{array}\right]_{q}\left[\begin{array}{c}
v-m \\
i
\end{array}\right]_{q} . }
\end{aligned}
$$

Finally apply the convention $\left[\begin{array}{l}a \\ b\end{array}\right]_{q}=0$ for integers with $b<0$ or $b>a$.

Note that $\operatorname{dim}(U \cap W) \geq k-t$ is equivalent to $\mathrm{d}_{\mathrm{s}}(U, W) \leq m-k+2 t$. The fact that the Grassmann graph is distance-regular implies:

Theorem 3. (Sphere-packing bound)[32, Theorem 6]

$$
A_{q}(v, d ; k) \leq \frac{\left[\begin{array}{l}
v \\
k
\end{array}\right]_{q}}{\sum_{i=0}^{\lfloor(d / 2-1) / 2\rfloor} q^{i^{2}}\left[\begin{array}{l}
k \\
i
\end{array}\right]_{q}\left[\begin{array}{c}
v-k \\
i
\end{array}\right]_{q}}
$$

We remark, that we can obtain the denominator of the formula of Theorem 3 by setting $m=k, 2 t=d / 2-1$ in Lemma 2 and applying $\left[\begin{array}{c}k \\ k-i\end{array}\right]_{q}=\left[\begin{array}{c}k \\ i\end{array}\right]_{q}$. The right hand side is symmetric with respect to orthogonal subspaces, i.e., the mapping $k \mapsto v-k$ leaves it invariant.

By defining a puncturing operation one can decrease the dimension of the ambient space and the codewords. Since the minimum distance decreases by at most two, we can iteratively puncture $d / 2-1$ times, so that $A_{q}(v, d ; k) \leq\left[\begin{array}{c}v-d / 2+1 \\ k-d / 2+1\end{array}\right]_{q}=\left[\begin{array}{c}v-d / 2+1 \\ v-k\end{array}\right]_{q}$ since $A_{q}\left(v^{\prime}, 2 ; k^{\prime}\right)=\left[\begin{array}{l}v^{\prime} \\ k^{\prime}\end{array}\right]_{q}$. Considering either the code or its orthogonal code gives:

Theorem 4. (Singleton bound)[32, Theorem 9]

$$
A_{q}(v, d ; k) \leq\left[\begin{array}{c}
v-d / 2+1 \\
\max \{k, v-k\}
\end{array}\right]_{q}
$$


Referring to [32] the authors of [29] state that even a relaxation of the Singleton bound is always stronger than the sphere packing bound for non-trivial codes. However, for $q=2, v=8, d=6$, and $k=4$, the sphere-packing bound gives an upper bound of $200787 / 451 \approx 445.20399$ while the Singleton bound gives an upper bound of $\left[\begin{array}{l}6 \\ 4\end{array}\right]_{2}=651$. For $q=2, v=8, d=4$, and $k=4$ it is just the other way round, i.e., the Singleton bound gives $\left[\begin{array}{l}7 \\ 3\end{array}\right]_{2}=11811$ and the sphere-packing bound gives $\left[\begin{array}{l}8 \\ 4\end{array}\right]_{2}=200787$. Examples for the latter case are easy to find. For $d=2$ both bounds coincide and for $d=4$ the Singleton bound is always stronger than the sphere-packing bound since $\left[\begin{array}{c}v-1 \\ k\end{array}\right]_{q}<\left[\begin{array}{l}v \\ k\end{array}\right]_{q}$. The asymptotic bounds [32, Corollaries 7 and 10], using normalized parameters, and [32, Figure 1] suggest that there is only a small range of parameters where the sphere-packing bound can be superior to the Singleton bound 1

Given an arbitrary metric space $X$, an anticode of diameter $e$ is a subset whose elements have pairwise distance at most $e$. Since the $q$-Johnson scheme is an association scheme the Anticode bound of Delsarte [11] can be applied. As a standalone argument we go along the lines of [2] and consider bounds for codes on transitive graphs. By double-counting the number of pairs $(a, g) \in A \cdot \operatorname{Aut}(\Gamma)$, where $g(a) \in B$, we obtain:

Lemma 3. [2, Lemma 1], cf. [3, Theorem 1'] Let $\Gamma=(V, E)$ be a graph that admits a transitive group of automorphisms $\operatorname{Aut}(\Gamma)$ and let $A, B$ be arbitrary subsets of the vertex set $V$. Then, there exists a group element $g \in \operatorname{Aut}(\Gamma)$ such that

$$
\frac{|g(A) \cap B|}{|B|} \geq \frac{|A|}{|V|}
$$

Corollary 1. [2, Corollary 1], cf. [3, Theorem 1] Let $\mathcal{C}_{D} \subseteq\left[\begin{array}{c}V \\ k\end{array}\right]$ be a code with (injection or graph) distances from $D=\left\{d_{1}, \ldots, d_{s}\right\} \subseteq\{1, \ldots, v\}$. Then, for an arbitrary subset $\mathcal{B} \subseteq\left[\begin{array}{l}V \\ k\end{array}\right]$ there exists a code $\mathcal{C}_{D}^{*}(\mathcal{B}) \subseteq \mathcal{B}$ with distances from $D$ such that

$$
\frac{\left|\mathcal{C}_{D}^{*}(\mathcal{B})\right|}{|\mathcal{B}|} \geq \frac{\left|\mathcal{C}_{D}\right|}{\left[\begin{array}{l}
v \\
k
\end{array}\right]_{q}}
$$

If $\mathcal{C}_{D} \subseteq\left[\begin{array}{c}V \\ k\end{array}\right]$ is a constant dimension code with minimum injection distance $d$, i.e., $D=\{d, \ldots, v\}$, and $\mathcal{B}$ is an anticode with diameter $d-1$, we have $\# \mathcal{C}_{D}^{*}(\mathcal{B})=1$, so that we obtain Delsarte's Anticode bound

$$
\# \mathcal{C}_{D} \leq \frac{\left[\begin{array}{l}
v \\
k
\end{array}\right]_{q}}{\# \mathcal{B}}
$$

The set of all elements of $\left[\begin{array}{c}V \\ k\end{array}\right]$ which contain a fixed $(k-d / 2+1)$-dimensional subspace is an anticode of diameter $d-2$ with $\left[\begin{array}{c}v-k+d / 2-1 \\ d / 2-1\end{array}\right]_{q}$ elements. By orthogonality, the set of all elements of $\left[\begin{array}{c}V \\ k\end{array}\right]$ which are contained in a fixed $(k+d / 2-1)$-dimensional subspace is also an anticode of diameter $d-2$ with $\left[\begin{array}{c}k+d / 2-1 \\ k\end{array}\right]_{q}=\left[\begin{array}{c}k+d / 2-1 \\ d / 2-1\end{array}\right]_{q}$ elements. Frankl and Wilson proved in [19, Theorem 1] that these anticodes have the largest possible size, which implies:

\footnotetext{
${ }^{1}$ By a tedious computation one can check that the sphere-packing bound is strictly tighter than the Singleton bound iff $q=2, v=2 k$ and $d=6$.
} 
Theorem 5. (Anticode bound)

$$
A_{q}(v, d ; k) \leq \frac{\left[\begin{array}{l}
v \\
k
\end{array}\right]_{q}}{\left[\begin{array}{c}
\max \{k, v-k\}+d / 2-1 \\
d / 2-1
\end{array}\right]_{q}}
$$

Using different arguments, Theorem 5 was proved in [42, Theorem 5.2] by Wang, Xing, Safavi-Naini in 2003. Codes that can achieve the (unrounded) value $\left[\begin{array}{l}v \\ k\end{array}\right]_{q} /\left[\begin{array}{c}\max \{k, v-k\}+d / 2-1 \\ d / 2-1\end{array}\right]_{q}$ are called Steiner structures. It is a well-known and seemingly very hard problem to decide whether a Steiner structure for $v=7, d=4$, and $k=3$ exists. For $q=2$ the best known bounds are $333 \leq A_{2}(7,4 ; 3) \leq 381$. Additionally it is known that a code attaining the upper bound can have automorphisms of at most order 2 , see [30]. So far, the only known Steiner structure corresponds to $A_{2}(13,4 ; 3)=1597245$ [9]. The reduction to Delsarte's Anticode bound can be found e.g. in [17, Theorem 1].

Since the sphere underlying the proof of Theorem 3 is also an anticode, Theorem 3 is implied by Theorem 5. For $d=2$ both bounds coincide. In [43, Section 4] Xia and Fu verified that the Anticode bound is always stronger than the Singleton bound for the ranges of parameters considered by us.

Mimicking a classical bound of Johnson on binary error-correcting codes with respect to the Hamming distance, see [28, Theorem 3] and also [41, Xia and Fu proved:

Theorem 6. (Johnson type bound I) [43, Theorem 2] If $\left(q^{k}-1\right)^{2}>\left(q^{v}-1\right)\left(q^{k-d / 2}-1\right)$, then

$$
A_{q}(v, d ; k) \leq \frac{\left(q^{k}-q^{k-d / 2}\right)\left(q^{v}-1\right)}{\left(q^{k}-1\right)^{2}-\left(q^{v}-1\right)\left(q^{k-d / 2}-1\right)} .
$$

However, the required condition of Theorem 6 is rather restrictive and can be simplified considerably.

Proposition 1. For $0 \leq k<v$, the bound in Theorem [6 is applicable iff $d=2 \min \{k, v-$ $k\}$ and $k \geq 1$. Then, it is equivalent to

$$
A_{q}(v, d ; k) \leq \frac{q^{v}-1}{q^{\min \{k, v-k\}}-1} .
$$

Proof. If $k=0$ we have $\left(q^{k}-1\right)^{2}=0$, so that we assume $k \geq 1$ in the following. If $k \leq v-k$ and $d \leq 2 k-2$, then

$$
\left(q^{v}-1\right)\left(q^{k-d / 2}-1\right) \geq\left(q^{2 k}-1\right)(q-1) \geq q^{2 k}-1 \stackrel{q \geq 2, k \geq 1}{>} q^{2 k}-2 q^{k}+1=\left(q^{k}-1\right)^{2} .
$$

If $k \geq v-k+1$ and $d \leq 2 v-2 k-2$, then

$$
\left(q^{v}-1\right)\left(q^{k-d / 2}-1\right) \geq\left(q^{v}-1\right)\left(q^{2}-1\right) \stackrel{q \geq 2, v \geq 1}{>}\left(q^{(v+1) / 2}-1\right)^{2} \geq\left(q^{k}-1\right)^{2} .
$$

If $d=2 \min \{k, v-k\}, q \geq 2$, and $k \geq 1$, then it can be easily checked that the condition of Theorem 6 is satisfied and we obtain the proposed formula after simplification. 
For $k=v$ Theorem 6 gives $A_{q}(v, d ; v) \leq 1$ which is trivially satisfied with equality. In Subsection 3.1 we will provide tighter upper bounds for the special case where $d=2 k$, i.e., partial spreads. Indeed, the bound stated in Proposition 1 corresponds to the most trivial upper bounds for partial spreads that is tight iff $k$ divides $v$, as we will see later on. So, due to orthogonality, Theorem 6 is dominated by the partial spread bounds discussed later on.

While the previously mentioned generalization of a classical bound of Johnson on binary error-correcting codes yields the rather weak Theorem 6, generalizing [28, Inequality (5)], see [43] yields a very strong upper bound:

Theorem 7. (Johnson type bound II) [43, Theorem 3], [17, Theorem 4,5]

$$
\begin{aligned}
& A_{q}(v, d ; k) \leq \frac{q^{v}-1}{q^{k}-1} A_{q}(v-1, d ; k-1) \\
& A_{q}(v, d ; k) \leq \frac{q^{v}-1}{q^{v-k}-1} A_{q}(v-1, d ; k)
\end{aligned}
$$

Note that for $d=2 k$ Inequality $(3)$ gives $A_{q}(v, 2 k ; k) \leq\left\lfloor\frac{q^{v}-1}{q^{k}-1}\right\rfloor$ since we have $A_{q}(v-$ $1,2 k ; k-1)=1$ by definition. Similarly, for $d=2(v-k)$, Inequality (4) gives $A_{q}(v, 2 v-$ $2 k ; k) \leq\left\lfloor\frac{q^{v}-1}{q^{v-k}-1}\right\rfloor$.

Some sources like [43, Theorem 3] list just Inequality 3 and omit Inequality 4 . This goes in line with the treatment of the classical Johnson type bound II for binary error-correcting codes, see e.g. [35, Theorem 4 on page 527], where the other bound is formulated as Problem (2) on page 528 with the hint that ones should be replaced by zeros. Analogously, we can consider orthogonal codes:

Proposition 2. Inequality (3) and Inequality (4) are equivalent using orthogonality, cf. [17, Section III, esp. Lemma 13].

Proof. We have

$$
\begin{aligned}
A_{q}(v, d ; k) & =A_{q}(v, d ; v-k) \stackrel{\sqrt[3]{3}}{\leq} \frac{q^{v}-1}{q^{v-k}-1} A_{q}(v-1, d ; v-k-1) \\
& =\frac{q^{v}-1}{q^{v-k}-1} A_{q}(v-1, d ; k)
\end{aligned}
$$

which is Inequality (4), and

$$
\begin{aligned}
A_{q}(v, d ; k) & =A_{q}(v, d ; v-k) \stackrel{\sqrt[4]{\leq}}{\leq} \frac{q^{v}-1}{q^{k}-1} A_{q}(v-1, d ; v-k) \\
& =\frac{q^{v}-1}{q^{k}-1} A_{q}(v-1, d ; k-1),
\end{aligned}
$$

which is Inequality (3). 
Of course, the bounds in Theorem 7 can be applied iteratively. In the classical Johnson space the optimal order of the corresponding inequalities is unclear, see e.g. [35, Research Problem 17.1]. Denoting the maximum size of a binary constant-weight block code of length $n$, Hamming distance $d$ and weight $k$ by $A(n, d, w)$, the two corresponding variants of the inequalities in Theorem 7 are $A(n, d, w) \leq\lfloor n / w \cdot A(n-1, d, w-1)\rfloor$ and $A(n, d, w) \leq\lfloor n /(n-w) \cdot A(n-1, d, w)\rfloor$. Applying the first bound yields

$$
A(28,8,13) \leq\lfloor 28 / 13 \cdot A(27,8,12)\rfloor \leq\lfloor 28 / 13 \cdot 10547\rfloor=22716
$$

while applying the second bound yields

$$
A(28,8,13) \leq\lfloor 28 / 15 \cdot A(27,8,13)\rfloor \leq\lfloor 28 / 15 \cdot 11981\rfloor=22364
$$

using the numerical bounds from

$$
\text { http://webfiles.portal.chalmers.se/s2/research/kit/bounds/cw.html, cf. [1]. }
$$

The authors of [17, 29] state that the optimal choice of Inequality (3) or Inequality (4) is unclear, too. However, this question is much easier to answer for constant dimension codes.

Proposition 3. For $k \leq v / 2$ we have

$$
\left\lfloor\frac{q^{v}-1}{q^{k}-1} A_{q}(v-1, d ; k-1)\right\rfloor \leq\left\lfloor\frac{q^{v}-1}{q^{v-k}-1} A_{q}(v-1, d ; k)\right\rfloor,
$$

where equality holds iff $v=2 k$.

Proof. By considering orthogonal codes we obtain equality for $v=2 k$. Now we assume $k<v / 2$ and show

$$
\frac{q^{v}-1}{q^{k}-1} A_{q}(v-1, d ; k-1)+1 \leq \frac{q^{v}-1}{q^{v-k}-1} A_{q}(v-1, d ; k),
$$

which implies the proposed statement. Considering the size of the LMRD code we can lower bound the right hand side of Inequality (5) to

$$
\frac{q^{v}-1}{q^{v-k}-1} A_{q}(v-1, d ; k) \geq \frac{q^{v}-1}{q^{v-k}} \cdot q^{(v-k-1)(k-d / 2+1)} .
$$

Since

$$
\frac{\left[\begin{array}{c}
v-1 \\
k-1
\end{array}\right]_{q}}{\left[\begin{array}{c}
v-k+d / 2-1 \\
d / 2-1
\end{array}\right]_{q}}=\frac{\prod_{i=1}^{k-1} \frac{q^{v-k+i}-1}{q^{i}-1}}{\prod_{i=1}^{d / 2-1} \frac{q^{v-k+i}-1}{q^{i}-1}} \leq \prod_{i=d / 2}^{k-1} \frac{q^{v-k+i}}{q^{i}-1}=q^{(v-k)(k-d / 2)} \prod_{i=d / 2}^{k-1} \frac{1}{1-q^{-i}}
$$

we can use the Anticode bound to upper bound the left hand side of Inequality (5) to

$$
\frac{q^{v}-1}{q^{k}-1} A_{q}(v-1, d ; k-1)+1 \leq \frac{q^{v}-1}{q^{k}-1} \cdot q^{(v-k)(k-d / 2)} \cdot \mu(k-1, d / 2, q)+1,
$$


where $\mu(a, b, q):=\prod_{i=b}^{a}\left(1-q^{-i}\right)^{-1}$. Thus, it suffices to verify

$$
\frac{q^{k-d / 2+1}}{q^{k}-1} \cdot \mu(k-1, d / 2, q)+\frac{1}{f} \leq 1
$$

where we have divided by

$$
f:=\frac{q^{v}-1}{q^{v-k}} \cdot q^{(v-k-1)(k-d / 2+1)}=\frac{q^{v}-1}{q} \cdot q^{(v-k-1)(k-d / 2)} .
$$

Since $d \geq 4$, we have $\mu(k-1, d / 2, q) \leq \prod_{i=2}^{\infty}\left(1-q^{-i}\right)^{-1} \leq \prod_{i=2}^{\infty}\left(1-2^{-i}\right)^{-1}<1.74$. Since $v \geq 4$ and $q \geq 2$, we have $\frac{1}{f} \leq \frac{2}{15}$. Since $k \geq 2$, we have $\frac{q^{k-d / 2+1}}{q^{k}-1} \leq \frac{q}{q^{2}-1}$, which is at most $\frac{3}{8}$ for $q \geq 3$. Thus, Inequality (6) is valid for all $q \geq 3$.

If $d \geq 6$ and $q=2$, then $\mu(k-1, d / 2, q) \leq \prod_{i=3}^{\infty}\left(1-2^{-i}\right)^{-1}<1.31$ and $\frac{q^{k-d / 2+1}}{q^{k}-1} \leq \frac{1}{3}$, so that Inequality (6) is satisfied.

In the remaining part of the proof we assume $d=4$ and $q=2$. If $k=2$, then $\mu(k-1, d / 2, q)=1$ and $\frac{q^{k-d / 2+1}}{q^{k}-1}=\frac{2}{3}$. If $k=3$, then $\mu(k-1, d / 2, q)=\frac{4}{3}$ and $\frac{q^{k-d / 2+1}}{q^{k}-1}=\frac{4}{7}$. If $k \geq 4$, then $\frac{q^{k-d / 2+1}}{q^{k}-1} \leq \frac{8}{15}, \mu(k-1, d / 2, q) \leq 1.74$, and $\frac{1}{f} \leq \frac{2}{255}$ due to $v \geq 2 k \geq 8$. Thus, Inequality (6) is valid in all cases.

Knowing the optimal choice between Inequality (3) and Inequality (4), we can iteratively apply Theorem 7 in an ideal way initially assuming $k \leq v / 2$ :

\section{Corollary 2. (Implication of the Johnson type bound II)}

$$
A_{q}(v, d ; k) \leq\left\lfloor\frac{q^{v}-1}{q^{k}-1}\left\lfloor\frac{q^{v-1}-1}{q^{k-1}-1}\left\lfloor\ldots\left\lfloor\frac{q^{v-k+d / 2+1}-1}{q^{d / 2+1}-1} A_{q}(v-k+d / 2, d ; d / 2)\right\rfloor \ldots\right\rfloor\right\rfloor\right\rfloor
$$

We remark that this upper bound is commonly stated in an explicit version, where $A_{q}(v-k+d / 2, d ; d / 2) \leq\left\lfloor\frac{q^{v-k+d / 2}-1}{q^{d / 2}-1}\right\rfloor$ is inserted, see e.g. [17, Theorem 6], [29, Theorem 7], and [43, Corollary 3]. However, currently much better bounds for partial spreads are available.

It is shown in [43] that the Johnson bound of Theorem 7 improves on the Anticode bound in Theorem 5, see also [6]. To be more precise, removing the floors in the upper bound of Corollary 2 and replacing $A_{q}(v-k+d / 2, d ; d / 2)$ by $\frac{q^{v-k+d / 2}-1}{q^{d / 2}-1}$ gives

$$
\prod_{i=0}^{k-d / 2} \frac{q^{v-i}-1}{q^{k-i}-1}=\frac{\prod_{i=0}^{k-1} \frac{q^{v-i}-1}{q^{k-i}-1}}{\prod_{i=k-d / 2+1}^{k-1} \frac{q^{v-i}-1}{q^{k-i}-1}}=\frac{\left[\begin{array}{c}
v \\
k
\end{array}\right]_{q}}{\left[\begin{array}{c}
v-k+d / 2-1 \\
d / 2-1
\end{array}\right]_{q}}
$$

which is the right hand side of the Anticode bound for $k \leq v-k$. So, all upper bounds mentioned so far are (weakly) dominated by Corollary 2 , if we additionally 
assume $k \leq v-k$. As a possible improvement [2, Theorem 3] was mentioned as [29, Theorem 8]. Here, we correct typos and give a slightly enlarged proof, thanks to a personal communication with Aydinian.

Theorem 8. [2, Theorem 3] For integers $0 \leq t<r \leq k, k-t \leq m \leq v$, and $t \leq v-m$ we have

$$
A_{q}(v, 2 r ; k) \leq \frac{\left[\begin{array}{l}
v \\
k
\end{array}\right]_{q} A_{q}(m, 2 r-2 t ; k-t)}{\sum_{i=0}^{t} q^{i(m+i-k)}\left[\begin{array}{c}
m \\
k-i
\end{array}\right]_{q}\left[\begin{array}{c}
v-m \\
i
\end{array}\right]_{q}} .
$$

Proof. Let $W$ be a fixed subspace with $\operatorname{dim}(W)=m$ and define

$$
\mathcal{B}=\left\{U \in\left[\begin{array}{c}
V \\
k
\end{array}\right] \mid \operatorname{dim}(U \cap W) \geq k-t\right\},
$$

so that $\# \mathcal{B}$ is given by Lemma 2. Consider a $\left(v, \# \mathcal{C}^{*}, d ; k\right)$ code $\mathcal{C}^{*} \subseteq \mathcal{B}$ and take $\mathcal{C}^{\prime}:=\mathcal{C}^{*} \cap W$ noting that the latter has a minimum distance of at least $2 r-2 t$. Two arbitrary codewords $U_{1} \neq U_{2} \in \mathcal{C}^{\prime}$ have distance $\mathrm{d}_{\mathrm{s}}\left(U_{1}, U_{2}\right) \geq 2 r-2 t+i+j$, where we write $\operatorname{dim}\left(U_{1}\right)=k-t+i$ and $\operatorname{dim}\left(U_{2}\right)=k-t+j$ for integers $0 \leq i, j \leq t$. Replacing each codeword of $\mathcal{C}^{\prime}$ by an arbitrary $k-t$-dimensional subspace, we obtain a cdc $\mathcal{C}$ with a minimum distance of at least $2 r-2 t$. Since $t<r$ we have $\# \mathcal{C}^{*}=\# \mathcal{C}^{\prime}=\# \mathcal{C}$, so that Corollary 1 gives the proposed upper bound.

As Theorem 8 has quite some degrees of freedom, we partially discuss the optimal choice of parameters. For $t=0$ and $m \leq v-1$, we obtain $A_{q}(v, d ; k) \leq\left[\begin{array}{c}v \\ k\end{array}\right]_{q} /\left[\begin{array}{c}m \\ k\end{array}\right]_{q} \cdot A_{q}(m, d ; k)$, which is the $(v-m)$-fold iteration of Inequality (4) of the Johnson bound (without rounding). Thus, $m=v-1$ is the best choice for $t=0$, yielding a bound that is equivalent to Inequality (4). For $t=1$ and $m=v-1$ the bound can be rewritten to $A_{q}(v, d ; k) \leq A_{q}(v-1, d-2 ; k-1)$, see the proof of Proposition 4. For $t>v-m$ the bound remains valid but is strictly weaker than for $t=v-m$. Choosing $m=v$ gives the trivial bound $A_{q}(v, 2 r ; k) \leq A_{q}(m, 2 r-2 t ; k-t)$. For the range of parameters $2 \leq q \leq 9$, $4 \leq v \leq 100$, limited facing nerve-jangling numerical pitfalls, and $4 \leq d \leq 2 k \leq v$, where $q$ is of course a prime power and $d$ is even, the situation is as follows. If $d \neq 2 k$, there are no proper improvements with respect to Theorem 7 , For the case $d=2 k$, i.e., partial spreads treated in the next subsection, we have some improvements compared to $\left\lfloor\left(q^{v}-1\right) /\left(q^{k}-1\right)\right\rfloor$ which is the most trivial bound for partial spreads. Within our numerical range, most of them are covered by the following proposition, where we apply Theorem 8 with $t=1$ and $m=v-1$ to $A_{q}(v, 2 k ; k)$. The other cases are due to the fact that Theorem 14 is tighter than Theorem 16 for larger values of $z$. In no case a proper improvement with respect to the tighter bounds from the next subsection emerged.

Proposition 4. For $w \geq 1$ and $k \geq q^{w}+3$ we have $A_{q}(2 k+w, 2 k ; k) \leq$

$$
\left\lfloor\frac{\left[\begin{array}{c}
2 k+w \\
k
\end{array}\right]_{q} A_{q}(2 k+w-1,2 k-2 ; k-1)}{\sum_{i=0}^{1} q^{i(k+w-1+i)}\left[\begin{array}{c}
2 k+w-1 \\
k-i
\end{array}\right]_{q}\left[\begin{array}{c}
(2 k+w)-(2 k+w-1) \\
i
\end{array}\right]}\right\rfloor<\left\lfloor\frac{q^{2 k+w}-1}{q^{k}-1}\right\rfloor=q^{k+w}+q^{w}
$$


Proof. Note that $k \geq q^{w}+3$ implies $w<k$. The left hand side simplifies to

$$
\frac{\left[\begin{array}{c}
2 k+w \\
k
\end{array}\right]_{q} A_{q}(2 k+w-1,2 k-2 ; k-1)}{\sum_{i=0}^{1} q^{i(k+w-1+i)}\left[\begin{array}{c}
2 k+w-1 \\
k-i
\end{array}\right]_{q}\left[\begin{array}{c}
(2 k+w)-(2 k+w-1) \\
i
\end{array}\right]_{q}}=A_{q}(2 k+w-1,2 k-2 ; k-1) .
$$

Then we apply Theorem 16 with $t=2, r=w+1$, and $z=\left[\begin{array}{c}w \\ 1\end{array}\right]_{q}-1$, which yields $A_{q}(2 k+w-1,2 k-2 ; k-1) \leq q^{k+w}+1+q^{w}-q<q^{k+w}+q^{w}$ for $k-1 \geq q^{w}+2$.

We remark that applying Theorem 14 and Theorem 16 directly is at least as good as the application of Theorem 8 with $t=1$ and $m=v-1$ for $d=2 k$.

The Delsarte linear programming bound for the $q$-Johnson scheme was obtained in [12. However, numerical computations indicate that it is not better than the Anticode bound, see [6]. For $d \neq 2 \min \{k, v-k\}$, i.e., the non-partial spread case, besides the stated bound only the following two specific bounds, based on extensive computer calculations, are known:

Theorem 9. [26, Theorem 1] $A_{2}(6,4 ; 3)=77$

Proposition 5. 24 $A_{2}(8,6 ; 4) \leq 272$

As the authors of [24] have observed, the Johnson bound of Theorem 7 does not improve upon Corollary 2 when applied to Theorem 9 or Proposition 5.

If we additionally restrict ourselves to constant dimension codes, that contain a lifted MRD code, another upper bound is known:

Theorem 10. [16, Theorem 10 and 11] Let $\mathcal{C} \subseteq\left[\begin{array}{c}\mathbb{F}_{q}^{v} \\ k\end{array}\right]$ be a constant dimension code, with $v \geq 2 k$ and minimum subspace distance $d$, that contains a lifted MRD code.

- If $d=2(k-1)$ and $k \geq 3$, then $\# \mathcal{C} \leq q^{2(v-k)}+A_{q}(v-k, 2(k-2) ; k-1)$;

- if $d=k$, where $k$ is even, then $\# \mathcal{C} \leq q^{(v-k)(k / 2+1)}+\left[\begin{array}{c}v-k \\ k / 2\end{array}\right]_{q} \frac{q^{v}-q^{v-k}}{q^{k}-q^{k / 2}}+A_{q}(v-k, k ; k)$.

\subsection{Upper bounds for partial spreads}

The case of constant dimension codes with maximum possible subspace distance $d=2 k$ is known under the name partial spreads. Counting points, i.e., 1-dimensional subspaces, in $\mathbb{F}_{q}^{v}$ and $\mathbb{F}_{q}^{k}$ gives the obvious upper bound $A_{q}(v, 2 k ; k) \leq\left[\begin{array}{l}v \\ 1\end{array}\right]_{q} /\left[\begin{array}{l}k \\ 1\end{array}\right]_{q}=\left(q^{v}-1\right) /\left(q^{k}-1\right)$. In the case of equality one speaks of spreads, for which a handy existence criterion is known from the work of Segre in 1964.

Theorem 11. 37, $\S V I] \mathbb{F}_{q}^{v}$ contains a spread if and only if $k$ is a divisor of $v$.

If $k$ is not a divisor of $v$, far better bounds are known including some recent improvements, which we will briefly summarize. For a more detailed treatment we refer to e.g. [27. The best known parametric construction was given by Beutelspacher in 1975: 
Theorem 12. [7] For positive integers $v, k$ satisfying $v=t k+r, t \geq 2$ and $1 \leq r \leq k-1$ we have $A_{q}(v, 2 k ; k) \geq 1+\sum_{i=1}^{t-1} q^{i k+r}=\frac{q^{v}-q^{k+r}+q^{k}-1}{q^{k}-1}$ with equality for $r=1$.

The determination of $A_{2}(v, 6 ; 3)$ for $v \equiv 2(\bmod 3)$ was achieved more than 30 years later in [14] and continued to $A_{2}(v, 2 k ; k)$ for $v \equiv 2(\bmod k)$ and arbitrary $k$ in [34]. Besides the parameters of $A_{2}(8+3 l, 6 ; 3)$, for $l \geq 0$, see [14] for an example showing $A_{2}(8,6 ; 3) \geq 34$, no partial spreads exceeding the lower bound from Theorem 12 are known.

For a long time the best known upper bound on $A_{q}(v, 2 k ; k)$ was the one obtained by Drake and Freeman in 1979:

Theorem 13. [13, Corollary 8] If $v=k t+r$ with $0<r<k$, then

$$
A_{q}(v, 2 k ; k) \leq \sum_{i=0}^{t-1} q^{i k+r}-\lfloor\theta\rfloor-1=q^{r} \cdot \frac{q^{k t}-1}{q^{k}-1}-\lfloor\theta\rfloor-1,
$$

where $2 \theta=\sqrt{1+4 q^{k}\left(q^{k}-q^{r}\right)}-\left(2 q^{k}-2 q^{r}+1\right)$.

Quite recently this bound has been generalized to:

Theorem 14. [33, Theorem 2.10] For integers $r \geq 1, t \geq 2, y \geq \max \{r, 2\}, z \geq 0$ with $\lambda=q^{y}, y \leq k, k=\left[\begin{array}{l}r \\ 1\end{array}\right]_{q}+1-z>r, v=k t+r$, and $l=\frac{q^{v}-k-q^{r}}{q^{k}-1}$, we have $A_{q}(v, 2 k ; k) \leq$ $l q^{k}+\left\lceil\lambda-\frac{1}{2}-\frac{1}{2} \sqrt{1+4 \lambda(\lambda-(z+y-1)(q-1)-1)}\right\rceil$.

The construction of Theorem 12 is asymptotically optimal for $k \gg r=v \bmod k$, as recently shown by Năstase and Sissokho:

Theorem 15. [36, Theorem 5] Suppose $v=t k+r$ with $t \geq 1$ and $0<r<k$. If $k>\left[\begin{array}{l}r \\ 1\end{array}\right]_{q}$ then $A_{q}(v, 2 k ; k)=1+\sum_{i=1}^{t-1} q^{i k+r}=\frac{q^{v}-q^{k+r}+q^{k}-1}{q^{k}-1}$.

Applying similar techniques, the result was generalized to $k \leq\left[\begin{array}{l}r \\ 1\end{array}\right]_{q}$ :

Theorem 16. [33, Theorem 2.9] For integers $r \geq 1, t \geq 2, u \geq 0$, and $0 \leq z \leq\left[\begin{array}{l}r \\ 1\end{array}\right]_{q} / 2$ with $k=\left[\begin{array}{l}r \\ 1\end{array}\right]_{q}+1-z+u>r$ we have $A_{q}(v, 2 k ; k) \leq l q^{k}+1+z(q-1)$, where $l=\frac{q^{v-k}-q^{r}}{q^{k}-1}$ and $v=k t+r$. [27.

Using Theorem 14 the restriction $z \leq\left[\begin{array}{l}r \\ 1\end{array}\right]_{q} / 2$ can be removed from Theorem 16 , see

Currently, Theorem 11, Theorem 14, and Theorem 16 constitute the tightest parametric bounds for $A_{q}(v, 2 k ; k)$. The only known improvements, by exactly one in every case, are given by the 21 specific bounds stated in [33], which are based on the linear programming method applied to projective $q^{k-1}$-divisible linear error-correcting codes over $\mathbb{F}_{q}$ with respect to the Hamming distance, see [27]. As this connection seemed to be overlooked before, it may not be improbable that more sophisticated methods from classical coding theory can improve further values, which then imply improved upper bounds for constant dimension codes via the Johnson bound of Theorem 7 . 


\section{The linkage construction revisited}

A very effective and widely applicable construction of constant dimension codes was stated by Gluesing-Luerssen and Troha:

Theorem 17. [22, Theorem 2.3], cf. [38, Corollary 39] Let $C_{i}$ be a $\left(v_{i}, N_{i}, d_{i} ; k\right)_{q}$ constant dimension code for $i=1,2$ and let $C_{r}$ be $a\left(k \times v_{2}, N_{r}, d_{r}\right)_{q}$ linear rank metric code. Then

$$
\left\{\tau^{-1}(\tau(U) \mid M): U \in C_{1}, M \in C_{r}\right\} \cup\left\{\tau^{-1}\left(0_{k \times v_{1}} \mid \tau(W)\right): W \in C_{2}\right\}
$$

is a $\left(v_{1}+v_{2}, N_{1} N_{R}+N_{2}, \min \left\{d_{1}, d_{2}, 2 d_{r}\right\} ; k\right)_{q}$ constant dimension code.

Here $A \mid B$ denotes the concatenation of two matrices with the same number of rows and $0_{m \times n}$ denotes the $m \times n$-matrix consisting entirely of zeros. The resulting code depends on the choice of the codes $C_{1}, C_{2}, C_{r}$ and their representatives within isomorphism classes, so that one typically obtains many isomorphism classes of codes with the same parameters.

We remark that [38, Theorem 37] corresponds to the weakened version of Theorem 17 where the codewords from the cdc $C_{2}$ are not taken into account, cf. [21, Theorem 5.1]. In [38, Corollary 39] Silberstein and (Horlemann-)Trautmann obtain the same lower bound, assuming $d_{1}=d_{2}=2 d_{r}$, which is indeed the optimal choice, and $3 k \leq v 2^{2}$

The main idea behind Theorem 17 is to consider two sets of codewords with disjoint pivot vectors across the two sets and to utilize the interplay between the rank and the subspace distance for a product type construction. Using Lemma 1 the restriction of the disjointness of the pivot vectors can be weakened, which gives the following improvement:

Theorem 18. Let $C_{i}$ be a $\left(v_{i}, N_{i}, d_{i} ; k\right)_{q}$ constant dimension code for $i=1,2, d \in 2 \mathbb{N}_{\geq 0}$ and let $C_{r}$ be $a\left(k \times\left(v_{2}-k+d / 2\right), N_{r}, d_{r}\right)_{q}$ linear rank metric code. Then

$$
\mathcal{C}=\left\{\tau^{-1}(\tau(U) \mid M): U \in C_{1}, M \in C_{r}\right\} \cup\left\{\tau^{-1}\left(0_{k \times\left(v_{1}-k+d / 2\right)} \mid \tau(W)\right): W \in C_{2}\right\}
$$

is a $\left(v_{1}+v_{2}-k+d / 2, N_{1} N_{R}+N_{2}, \min \left\{d_{1}, d_{2}, 2 d_{r}, d\right\} ; k\right)_{q}$ constant dimension code.

Proof. The dimension of the ambient space and the codewords of $\mathcal{C}$ directly follow from the construction. Since the constructed matrices all are in rref and pairwise distinct, $\mathcal{C}$ is well defined and we have $\# \mathcal{C}=N_{1} N_{R}+N_{2}$. It remains to lower bound the minimum subspace distance of $\mathcal{C}$.

Let $A, C \in C_{1}$ and $B, D \in C_{r}$. If $A \neq C$, we have

$$
\begin{array}{r}
\mathrm{d}_{\mathrm{s}}\left(\tau^{-1}((\tau(A) \mid B)), \tau^{-1}((\tau(C) \mid D))\right)=2\left(\operatorname{rk}\left(\begin{array}{cc}
\tau(A) & B \\
\tau(C) & D
\end{array}\right)-k\right) \\
\geq 2\left(\operatorname{rk}\left(\begin{array}{c}
\tau(A) \\
\tau(C)
\end{array}\right)-k\right)=\mathrm{d}_{\mathrm{s}}(A, C) \geq d_{1}
\end{array}
$$

\footnotetext{
${ }^{2}$ It can be verified that for $2 k \leq v \leq 3 k-1$ the optimal choice of $\Delta$ in [38, Corollary39] is given by $\Delta=v-k$. In that case the construction is essentially the union of an LMRD code with an $\left(v-k, \# \mathcal{C}^{\prime}, d ; k\right)_{q}$ code $\mathcal{C}^{\prime}$. Note that for $v-k<\Delta \leq v$ the constructed code is an embedded $\left(\Delta, \# \mathcal{C}^{\prime}, d ; k\right)_{q}$ code $\mathcal{C}^{\prime}$.
} 
using Equation (1) in the first step. If $A=C$ but $B \neq D$, we have

$$
\begin{aligned}
& \mathrm{d}_{\mathrm{s}}\left(\tau^{-1}((\tau(A) \mid B)), \tau^{-1}((\tau(C) \mid D))\right)=2\left(\operatorname{rk}\left(\begin{array}{cc}
\tau(A) & B \\
\tau(C) & D
\end{array}\right)-k\right) \\
& \geq 2\left(\operatorname{rk}\left(\begin{array}{cc}
\tau(A) & B \\
0 & D-B
\end{array}\right)-k\right)=2(k+\operatorname{rk}(D-B)-k) \geq 2 d_{r} .
\end{aligned}
$$

For $A^{\prime} \neq C^{\prime} \in C_{2}$ applying Equation (1) gives

$$
\mathrm{d}_{\mathrm{s}}\left(\tau^{-1}\left(0_{k \times\left(v_{1}-k+d / 2\right)} \mid \tau\left(A^{\prime}\right)\right), \tau^{-1}\left(0_{k \times\left(v_{1}-k+d / 2\right)} \mid \tau\left(C^{\prime}\right)\right)\right)=\mathrm{d}_{\mathrm{s}}\left(A^{\prime}, C^{\prime}\right) \geq d_{2} .
$$

Last, for two codewords $U \in\left\{\tau^{-1}(\tau(U) \mid M) \mid U \in C_{1}, M \in C_{r}\right\}$ and $W \in$ $\left\{\tau^{-1}\left(0_{k \times\left(v_{1}-k+d / 2\right)} \mid \tau(W)\right) \mid W \in C_{2}\right\}$, we can use the shape of the pivot vectors and apply Lemma 1. The pivot vector $p(U)$ has its $k$ ones in the first $v_{1}$ positions and the pivot vector $p(W)$ has its $k$ ones not in the first $v_{1}-k+d / 2$ positions, so that the ones can coincide at most at the positions $\left\{v_{1}-k+d / 2+1, \ldots, v_{1}\right\}$. Thus, $\mathrm{d}_{\mathrm{h}}(p(U), p(W)) \geq k-(k-d / 2)+k-(k-d / 2)=d$. Lemma 1 then gives $\mathrm{d}_{\mathrm{s}}(U, W) \geq d$.

An example where Theorem 18 yields a larger code than Theorem 17 is e.g. given for the parameters $q=2, v=7, d=4$, and $k=3$. In order to apply Theorem 17 we have to choose $v_{1}+v_{2}=7,3 \leq v_{1} \leq 4$, and $3 \leq v_{2} \leq 4$. For $v_{1}=3$ we obtain $\# C_{1} \leq A_{2}(3,4 ; 3)=1$ and $\# C_{2} \leq A_{2}(4,4 ; 3)=1$. Since the size of the rank metric code is bounded by $\left[2^{4(3-2+1)}\right]=2^{8}$, the constructed code has a size of at most $1 \cdot 2^{8}+1=257$. For $v_{1}=4$ the roles of $C_{1}$ and $C_{2}$ interchange. Since the size of the rank metric code is bounded by $\left[2^{3(3-2+1)}\right]=2^{6}$, the constructed code has a size of at most $1 \cdot 2^{6}+1=65$. In Theorem 18 we can choose $d=4$, so that we can drop one column of the zero matrix preceding the matrices of the second set of codewords, i.e., $v_{1}+v_{2}=7+1=8$. Choosing $v_{1}=3$ and $v_{2}=5$ we can achieve $\# C_{1}=A_{2}(3,4 ; 3)=1$ and $\# C_{2}=A_{2}(5,4 ; 3)=9$. Since the size of the rank metric code can attain $\left[2^{4(3-2+1)}\right]=2^{8}$ we can construct a code of size $1 \cdot 2^{8}+9=265$. While for these parameters sill larger codes are known, the situation significantly changes in general. Considering the range of parameters $2 \leq q \leq 9$, $4 \leq v \leq 19$, and $4 \leq d \leq 2 k \leq v$, where $q$ is of course a prime power and $d$ is even, Theorem 17 provides the best known lower bound for $A_{q}(v, d ; k)$ in $41.8 \%$ of the cases, while Theorem 18 provides the best known lower bound in $65.6 \%$ of the cases. Since the sizes of both constructions can coincide, the sum of both fractions gives more than $100 \%$. In just $34.4 \%$ of the cases strictly superior constructions are known compared to Theorem 18, where most of them arose from the so-called Echelon-Ferrers construction or one of their variants, see [23] and the corresponding webpage $3^{3}$

If one is interested in codes of large size, then one should choose the parameters $d_{1}, d_{2}$, $d_{r}$, and $d$, in Theorem 18, as small as possible in order to maximize the sizes $N_{1}, N_{2}$, and $N_{r}$, i.e., we can assume $d_{1}=d_{2}=2 d_{r}=d$. Moreover, the codes $C_{1}, C_{2}$, and $C_{r}$ should have the maximum possible size with respect to their specified parameters. For

\footnotetext{
${ }^{3}$ Entries of type improved_linkage(m) correspond to Corollary 4 with $m$ chosen as parameter.
} 
$C_{r}$ the maximum possible size is $M\left(q, k, v_{2}+d / 2, d\right)$ and for $C_{i}$ the maximum possible size is $A_{q}\left(v_{1}, d ; k\right)$, where $i=1,2$.

Corollary 3. For positive integers $k \leq \min \left\{v_{1}, v_{2}\right\}$ and $d \equiv 0(\bmod 2)$ we have $A_{q}\left(v_{1}+\right.$ $\left.v_{2}-k+d / 2, d ; k\right) \geq A_{q}\left(v_{1}, d ; k\right) \cdot M\left(q, k, v_{2}+d / 2, d\right)+A_{q}\left(v_{2}, d ; k\right)$.

Instead of $A_{q}\left(v_{1}, d ; k\right)$ or $A_{q}\left(v_{2}, d ; k\right)$ we may also insert any lower bound of these commonly unknown values. By a variable transformation we obtain:

Corollary 4. For positive integers $k \leq m \leq v-d / 2$ and $d \equiv 0(\bmod 2)$ we have $A_{q}(v, d ; k) \geq A_{q}(m, d ; k) \cdot M(q, k, v-m+k, d)+A_{q}(v-m+k-d / 2, d ; k)$.

For the parameters of spreads the optimal choice of the parameter $m$ in Corollary 4 can be determined analytically:

Lemma 4. If $d=2 k$ and $k$ divides $v$, then Corollary 4 gives $A_{q}(v, d ; k) \geq \frac{q^{v}-1}{q^{k}-1}$ for all $m=k, 2 k, \ldots, v-k$ and smaller sizes otherwise.

Proof. Using $A_{q}\left(v^{\prime}, 2 k ; k\right)=\left(q^{v^{\prime}}-1\right) /\left(q^{k}-1\right)$ for all integers $v^{\prime}$ being divisible by $k$, we obtain

$$
\begin{aligned}
A_{q}(v, d ; k) & \geq A_{q}(m, d ; k) \cdot M(q, k, v-m+k, 2 k)+A_{q}(v-m, 2 k ; k) \\
& =\frac{q^{m}-1}{q^{k}-1} \cdot q^{v-m}+\frac{q^{v-m}-1}{q^{k}-1}=\frac{q^{v}-1}{q^{k}-1}
\end{aligned}
$$

if $k$ divides $m$. Otherwise, $A_{q}(m, 2 k ; k) \leq\left(q^{m}-1\right) /\left(q^{k}-1\right)-1$ gives a lower bound.

We remark that the tightest implications of Corollary 4 can be evaluated by dynamic programming. To this end we consider fixed parameters $q, d, k$ and use the abbreviations $a(n):=A_{q}(n, d ; k)$ and $b(n):=M(q, k, n+k, d)$ for integers $n$, so that the inequality of Corollary 4 reads

$$
a(v) \geq a(m) \cdot b(v-m)+a(v-m+k-d / 2) .
$$

For a given maximal value $v$ we initialize the values $a(n)$ for $1 \leq n \leq v$ by the best known lower bounds for $A_{q}(n, d ; k)$ from other constructions. Then we loop over $n$ from $k$ to $v$ and eventually replace $a(n)$ by

$$
\max \{a(m) \cdot b(n-m)+a(n-m+k-d / 2) \mid k \leq m \leq n-d / 2\} .
$$

By an arithmetic progression we can use (7) in order to obtain a lower bound for $a(v)=A_{q}(v, d ; k)$ given just two initial $a(i)$-values.

Proposition 6. For positive integers $k \leq v_{0}, 2 s \geq d$, and $l \geq 0$, we have

$$
a\left(v_{0}+l s\right) \geq a\left(v_{0}\right) \cdot b(s)^{l}+a(s-d / 2+k)\left[\begin{array}{l}
l \\
1
\end{array}\right]_{b(s)} .
$$

If additionally, $v_{0} \geq 2 k-d / 2$ and $k \geq d / 2$, then we have

$$
a\left(v_{0}+l s\right) \geq a(s+k-d / 2) \cdot\left(q^{k-d / 2+1}\right)^{n_{0}-k+d / 2}\left[\begin{array}{l}
l \\
1
\end{array}\right]_{q^{s(k-d / 2+1)}}+a\left(v_{0}\right) .
$$


Proof. Using Inequality (7) with $v=v_{0}+l s$ and $m=v_{0}+(l-1) s$ gives

$$
a\left(v_{0}+l s\right) \geq a\left(v_{0}+(l-1) s\right) \cdot b(s)+a(s+k-d / 2) .
$$

By induction, we obtain

$$
a\left(v_{0}+l s\right) \geq a\left(v_{0}+(l-i) s\right) \cdot b(s)^{i}+a(s+k-d / 2)\left[\begin{array}{l}
i \\
1
\end{array}\right]_{b(s)}
$$

for all $0 \leq i \leq l$.

For the second part, applying Inequality (7) with $v=v_{0}+l s$ and $m=s+k-d / 2$ gives

$$
a\left(v_{0}+l s\right) \geq a(s+k-d / 2) \cdot b\left(v_{0}+(l-1) s-k+d / 2\right)+a\left(v_{0}+(l-1) s\right) .
$$

By induction, we obtain

$$
a\left(v_{0}+l s\right) \geq a(s+k-d / 2) \cdot \sum_{j=1}^{i} b\left(v_{0}+(l-j) s-k+d / 2\right)+a\left(v_{0}+(l-i) s\right)
$$

for all $0 \leq i \leq l$.

If $v_{0} \geq 2 k-d / 2$ and $k \geq d / 2$, then

$$
b\left(v_{0}+(l-j) s-k+d / 2\right)=\left(q^{k-d / 2+1}\right)^{v_{0}+(l-j) s-k+d / 2},
$$

so that

$$
\begin{array}{r}
\sum_{j=1}^{l} b\left(v_{0}+(l-j) s-k+d / 2\right)=\sum_{j=1}^{l}\left(q^{k-d / 2+1}\right)^{v_{0}+(l-j) s-k+d / 2}= \\
\left(q^{k-d / 2+1}\right)^{v_{0}-k+d / 2} \sum_{r=0}^{l-1}\left(q^{s(k-d / 2+1)}\right)^{r}=\left(q^{k-d / 2+1}\right)^{v_{0}-k+d / 2}\left[\begin{array}{l}
l \\
1
\end{array}\right]_{q^{s(k-d / 2+1)}} .
\end{array}
$$

Example 1. Using $A_{2}(13,4 ; 3)=1597245$ [9] and $A_{2}(7,4 ; 3) \geq 333$ [23], applying Proposition 6 with $s=6$ gives

$$
A_{2}(13+6 l, 4 ; 3) \geq 4096^{l} \cdot 1597245+333 \cdot \frac{4096^{l}-1}{4095}
$$

and

$$
A_{2}(13+6 l, 4 ; 3) \geq 333 \cdot 16777216 \cdot \frac{4096^{l}-1}{4095}+1597245
$$

for all $l \geq 0$.

In the next section we will see that the first lower bound almost meets the Anticode bound.

We remark that Theorem 18 can be easily generalized to a construction based on a union of $m \geq 2$ sets of codewords. 
Corollary 5. For positive integers $k, m$, and $i=1, \ldots, m$ let

- $C_{i}$ be an $\left(v_{i}, N_{i}, d_{i} ; k\right)_{q}$ constant dimension code,

- $\delta_{i} \in \mathbb{N}_{\geq 0}, \delta_{m}=0$,

- $C_{i}^{R}$ be a $\left(k \times v_{i}^{R}, N_{i}^{R}, d_{i}^{R}\right)_{q}$ linear rank metric code, where $v_{i}^{R}=\sum_{j=1}^{i-1}\left(v_{j}-\delta_{j}\right)$ and $i \neq 1$,

- $C_{1}^{R}=\emptyset, v_{1}^{R}=0, N_{1}^{R}=1$, and $d_{1}^{R}=\infty$.

Then

$$
\bigcup_{i=1}^{m}\left\{\tau^{-1}\left(0_{k \times\left(v-v_{i}-v_{i}^{R}\right)}\left|\tau\left(U_{i}\right)\right| M_{i}\right): U_{i} \in C_{i}, M_{i} \in C_{i}^{R}\right\}
$$

is a $(v, N, d ; k)_{q}$ constant dimension code with

- $v=\sum_{i=1}^{m}\left(v_{i}-\delta_{i}\right)$,

- $N=\sum_{i=1}^{m} N_{i} \cdot N_{i}^{R}$, and

- $d=\min \left\{d_{i}, 2 d_{i}^{R}, 2\left(k-\delta_{i}\right) \mid i=1, \ldots, m\right\}$.

Proof. We prove by inductively applying Theorem $18 m-1$ times. Denote

$$
\tilde{C}_{i}:=\left\{\tau^{-1}\left(0_{k \times\left(v-v_{i}-v_{i}^{R}\right)}\left|\tau\left(U_{i}\right)\right| M_{i}\right): U_{i} \in C_{i}, M_{i} \in C_{i}^{R}\right\}
$$

for $i=1, \ldots, m$, i.e., $\tilde{C}_{i}$ is a padded $\left(v_{i}+v_{i}^{R}, N_{i} \cdot N_{i}^{R}, \min \left\{d_{i}, 2 d_{i}^{R}\right\} ; k\right)_{q}$ constant dimension code. Applying Theorem 18 for $\tilde{C}_{1}$ and $\tilde{C}_{2}$ with $d=2\left(k-\delta_{1}\right)$ yields a $\left(v_{1}+v_{2}-\delta_{1}, N_{1}+\right.$ $\left.N_{2} \cdot N_{2}^{R}, \min \left\{d_{1}, d_{2}, 2 d_{2}^{R}, 2\left(k-\delta_{1}\right)\right\} ; k\right)_{q}$ constant dimension code. If the first $m^{\prime}$ codes, $\tilde{C}_{1}, \ldots, \tilde{C}_{m^{\prime}}$ yield an $\left(\sum_{i=1}^{m^{\prime}}\left(v_{i}-\delta_{i}\right)+\delta_{m^{\prime}}, \sum_{i=1}^{m^{\prime}} N_{i} \cdot N_{i}^{R}, \min \left\{d_{i}, 2 d_{i}^{R}, 2\left(k-\delta_{i}\right) \mid i=\right.\right.$ $\left.\left.1, \ldots, m^{\prime}\right\} ; k\right)_{q}$ constant dimension code $\tilde{C}_{1, \ldots, m^{\prime}}$, then performing Theorem 18 for this code and $\tilde{C}_{m^{\prime}+1}$ with $d=2\left(k-\delta_{m^{\prime}}\right)$ yields an $\left(\sum_{i=1}^{m^{\prime}}\left(v_{i}-\delta_{i}\right)+\delta_{m^{\prime}}+n_{2}-\delta_{m^{\prime}}, \sum_{i=1}^{m^{\prime}} N_{i}\right.$. $\left.N_{i}^{R}+N_{m^{\prime}+1} \cdot N_{m^{\prime}+1}^{R}, \min \left\{d_{i}, 2 d_{i}^{R}, 2\left(k-\delta_{i}\right) \mid i=1, \ldots, m^{\prime}+1\right\} ; k\right)_{q}$ constant dimension code.

Since the proof uses multiple applications of Theorem 18 this code can be found by the dynamic programming approach based on Theorem 18, i.e., Corollary 5 is redundant. However, it can be used to prove:

Corollary 6 (cf. [22, Theorem 4.6]). Let $C^{R}$ be an $\left(k \times v_{1}+v_{2}, d\right)_{q}$ linear MRD code, where $k \leq v_{i}$, for $i=1,2$ and let $C_{i}$ be an $\left(v_{i-2}, N_{i}, 2 d ; k\right)_{q}$ constant dimension codes for $i=3,4$. Then

$$
\begin{aligned}
& \left\{\tau^{-1}\left(I_{k \times k} \mid A\right) \mid A \in C^{R}\right\} \\
\cup & \left\{\tau^{-1}\left(0_{k \times k}|\tau(A)| 0_{k \times v_{2}}\right) \mid A \in C_{3}\right\} \\
\cup & \left\{\tau^{-1}\left(0_{k \times k}\left|0_{k \times v_{1}}\right| \tau(A)\right) \mid A \in C_{4}\right\}
\end{aligned}
$$

is a $\left(v_{1}+v_{2}+k, q^{\left(v_{1}+v_{2}\right)(k-d+1)}+N_{3}+N_{4}, 2 \min \{d, k\} ; k\right)_{q}$ constant dimension code. Note that $k<d$ implies $N_{3}, N_{4} \leq 1$. 
Proof. Applying Corollary 5 with

- $m=3$

- $\bar{C}_{1}=C_{4}, \bar{C}_{2}=C_{3}$,

- $\bar{C}_{3}=\left\{\tau^{-1}\left(I_{k \times k}\right)\right\}$ (i.e., an $(k, 1, \infty ; k)_{q}$ constant dimension code)

- $\delta_{1}=\delta_{2}=\delta_{3}=0$

- $\bar{C}_{1}^{R}=\emptyset$

- $\bar{C}_{2}^{R}=\left\{0_{k \times v_{2}}\right\}$ (i.e., an $\left(k \times v_{2}, 1, \infty\right)_{q}$ rank metric code)

- $\bar{C}_{3}^{R}$ an $\left(k \times\left(v_{1}+v_{2}, d\right)\right)_{q}$ MRD code

yields an $\left(v_{1}+v_{2}+k, N_{4}+N_{3}+q^{\left(v_{1}+v_{2}\right)(k-d+1)}, 2 \min \{d, k\} ; k\right)_{q}$ constant dimension code:

$$
\begin{aligned}
& \left\{\tau^{-1}\left(I_{k \times k} \mid M_{3}\right): M_{3} \in \bar{C}_{3}^{R}\right\} \\
\cup & \left\{\tau^{-1}\left(0_{k \times k}\left|\tau\left(U_{2}\right)\right| 0_{k \times v_{2}}\right): U_{2} \in C_{3}\right\} \\
\cup & \left\{\tau^{-1}\left(0_{k \times\left(v_{1}+k\right)} \mid \tau\left(U_{1}\right)\right): U_{1} \in C_{4}\right\}
\end{aligned}
$$

We remark that $\left\{(A \mid B): A \in C_{1}^{R}, B \in C_{2}^{R}\right\}$ is a $\left(k \times\left(v_{1}+v_{2}\right), d\right)_{q}$ linear MRD code, since each codeword has $\operatorname{rk}(A \mid B) \geq \operatorname{rk}(A) \geq d$. The other direction is not necessarily true, e.g., $\left(\begin{array}{c}I_{k-1} \\ 0\end{array} 0|\ldots| 0 \mid w\right)$, where $w$ is a non-zero column, cannot be split in two matrices $\left(I_{k-1}|0| \ldots \mid 0\right)$ and $(0|\ldots| 0 \mid w)$ both having rank distance at least $d$ for $d \geq 2$. Hence, this corollary constructs codes of the same size as Theorem 4.6 in [22] but these codes are not necessarily equal.

\section{Asymptotic bounds}

Kötter and Kschischang have stated the bounds

$$
1<q^{-k(v-k)} \cdot\left[\begin{array}{l}
v \\
k
\end{array}\right]_{q}<4
$$

for $0<k<v$ in [32, Lemma 4] for the $q$-binomial coefficients. They used this result in order to prove that the lifted MRD codes, they spoke about linearized polynomials, have at least a size of a quarter of the Singleton bound if $v$ tends to infinity. Actually, they have derived a more refined bound, which can best expressed using the so called $q$-Pochhammer symbol $(a ; q)_{n}:=\prod_{i=0}^{n-1}\left(1-a q^{i}\right)$ specializing to $(1 / q ; 1 / q)_{n}=\prod_{i=1}^{n}\left(1-1 / q^{i}\right)$ :

$$
1 \leq \frac{\left[\begin{array}{l}
v \\
k
\end{array}\right]_{q}}{q^{k(v-k)}} \leq \frac{1}{(1 / q ; 1 / q)_{k}} \leq \frac{1}{(1 / q ; 1 / q)_{\infty}} \leq \frac{1}{(1 / 2 ; 1 / 2)_{\infty}} \approx 3.4627,
$$

where $(1 / q ; 1 / q)_{\infty}$ denotes $\lim _{n \rightarrow \infty}(1 / q ; 1 / q)_{n}$, cf. the estimation for the Anticode bound in the proof of Proposition 3 . The sequence $(1 / q ; 1 / q)_{\infty}$ is monotonically increasing with $q$ and approaches $(q-1) / q$ for large $q$, see e.g. [32] and [29] for some numerical values. 
Lemma 5. For each $b \in \mathbb{N}_{\geq 0}$ we have $\lim _{a \rightarrow \infty} \frac{\left[\begin{array}{c}a+b \\ b\end{array}\right]_{q}}{q^{a b}}=\frac{1}{(1 / q ; 1 / q)_{b}}$.

Proof. Plugging in the definition of the $q$-binomial coefficient, we obtain

$$
\lim _{a \rightarrow \infty} \frac{\left[\begin{array}{c}
a+b \\
b
\end{array}\right]_{q}}{q^{a b}}=\lim _{a \rightarrow \infty} \frac{\prod_{i=1}^{b} \frac{q^{a+i}-1}{q^{i}-1}}{q^{a b}}=\prod_{i=1}^{b} \frac{q^{i}}{q^{i}-1}=\prod_{i=1}^{b} \frac{1}{1-1 / q^{i}}=\frac{1}{(1 / q ; 1 / q)_{b}} .
$$

Using this asymptotic result we can compare the size of the lifted MRD codes to the Singleton and the Anticode bound.

Proposition 7. For $k \leq v-k$ the ratio of the size of an LMRD code divided by the size of the Singleton bound converges for $v \rightarrow \infty$ monotonically decreasing to $(1 / q ; 1 / q)_{k-d / 2+1} \geq$ $(1 / 2 ; 1 / 2)_{\infty}>0.288788$.

Proof. Setting $z=k-d / 2+1$ and $s=v-k$ the ratio is given by $g(s):=\frac{q^{s z}}{\left[\begin{array}{c}s+z \\ z\end{array}\right]_{q}}$, so that Lemma 5 gives the proposed limit. The sequence is monotonically decreasing, since we have $0 \leq z-1<z \leq s+z$ and

$$
\frac{g(s)}{g(s+1)}=\frac{q^{s z}\left[{ }_{z}^{s+1+z}\right]_{q}}{\left[\begin{array}{c}
s+z \\
z
\end{array}\right]_{q} q^{(s+1) z}}=\frac{q^{z}\left[\begin{array}{c}
s+z \\
z
\end{array}\right]_{q}+\left[\begin{array}{c}
s+z \\
z-1
\end{array}\right]_{q}}{\left[\begin{array}{c}
s+z \\
z
\end{array}\right]_{q} q^{z}}=1+\frac{\left[\begin{array}{c}
s+z \\
z-1
\end{array}\right]_{q}}{\left[\begin{array}{c}
s+z \\
z
\end{array}\right]_{q} q^{z}}>1 .
$$

Proposition 8. For $k \leq v-k$ the ratio of the size of an LMRD code divided by the size of the Anticode bound converges for $v \rightarrow \infty$ monotonically decreasing to $\frac{(1 / q ; 1 / q)_{k}}{(1 / q ; 1 / q)_{d / 2-1}} \geq$ $\frac{q}{q-1} \cdot(1 / q ; 1 / q)_{k} \geq 2 \cdot(1 / 2 ; 1 / 2)_{\infty}>0.577576$.

Proof. The LMRD code has cardinality $q^{(v-k)(k-d / 2+1)}$ and the Anticode bound is $\left[\begin{array}{l}v \\ k\end{array}\right]_{q} /\left[\begin{array}{c}v-k+d / 2-1 \\ d / 2-1\end{array}\right]_{q}$. From Lemma 5 we conclude

$$
\lim _{v \rightarrow \infty} \frac{\left[\begin{array}{c}
(v-k)+k \\
k
\end{array}\right]_{q}}{q^{(v-k) k}}=\frac{1}{(1 / q ; 1 / q)_{k}} \text { and } \lim _{v \rightarrow \infty} \frac{\left[\begin{array}{c}
(v-k)+(d / 2-1) \\
d / 2-1
\end{array}\right]_{q}}{q^{(v-k)(d / 2-1)}}=\frac{1}{(1 / q ; 1 / q)_{d / 2-1}},
$$

so that the limit follows. The subsequent inequalities follow from $d \geq 4$, the monotonicity of $(1 / q ; 1 / q)_{n}$, and $q \geq 2$.

It remains to show the monotonicity of the sequence

$$
g(v):=\frac{q^{(v-k)(k-d / 2+1)}\left[\begin{array}{c}
v-k+d / 2-1 \\
d / 2-1
\end{array}\right]_{q}}{\left[\begin{array}{l}
v \\
k
\end{array}\right]_{q}}
$$


Using the abbreviation $s=v-k$ we define

$$
f(x):=\frac{\left[\begin{array}{c}
s+x \\
s+1
\end{array}\right]_{q}}{q^{x}\left[\begin{array}{c}
s+x \\
s
\end{array}\right]_{q}}=\frac{\prod_{i=1}^{s+1} \frac{q^{x-1+i}-1}{q^{i}-1}}{q^{x} \prod_{i=1}^{s} \frac{q^{x+i}-1}{q^{i}-1}}=\frac{\frac{q^{x}-1}{q^{s+1}-1}}{q^{x}}=\frac{1-q^{-x}}{q^{s+1}-1}
$$

and observe that $f$ is strictly monotonically increasing in $x$, so that $f(k)>f(d / 2-1)$. Using routine manipulations of $q$-binomial coefficients we compute

$$
\frac{g(v)}{g(v+1)}=(1+f(k)) \cdot(1+f(d / 2-1))^{-1}>1 .
$$

In other words the ratio between the best known lower bound and the best known upper bound for constant dimension codes is strictly greater than 0.577576 for all parameters and the most challenging parameters are given by $q=2, d=4$, and $k=\lfloor v / 2\rfloor$.

Replacing the Anticode bound by the Johnson bound of Theorem 2 does not change the limit behavior of Proposition 8 when $v$ tends to infinity. As stated above, we obtain the Anticode bound if we remove the floors in Corollary 2 and replace $A_{q}(v-k+d / 2, d ; d / 2)$ by $\frac{q^{v-k+d / 2}-1}{q^{d / 2}-1}$. First we consider the latter weakening. Applying the lower bound of Theorem 12 for $A_{q}\left(v^{\prime}, 2 k^{\prime} ; k^{\prime}\right)$, where $v^{\prime}=t k^{\prime}+r$ with $1 \leq r \leq k^{\prime}-1$, we consider

$$
\frac{q^{v^{\prime}}-q^{k^{\prime}+r}+q^{k^{\prime}}-1}{q^{k^{\prime}}-1} / \frac{q^{v^{\prime}}-1}{q^{k^{\prime}}-1}=1-\frac{q^{k^{\prime}} \cdot\left(q^{r}-1\right)}{q^{v^{\prime}}-1}
$$

If $v^{\prime} \geq 3 k^{\prime}$, then the subtrahend on the right hand side is at most $\frac{q^{v^{\prime} / 3} \cdot\left(\cdot v^{v^{\prime} / 3}-1\right)}{q^{v^{\prime}}-1}$. Otherwise we have $2 k^{\prime} \leq v^{\prime}<3 k^{\prime}$, so that $v^{\prime}=2 k^{\prime}+r$. Since $q^{k^{\prime}} \cdot\left(q^{r}-1\right) \cdot\left(q^{k^{\prime}}+1\right)=q^{2 k^{\prime}+r}-q^{2 k^{\prime}}+$ $q^{k^{\prime}+r}-q^{k^{\prime}} \leq q^{2 k^{\prime}+r}-1$ the subtrahend on the right hand side is at most $1 /\left(q^{v^{\prime} / 3}+1\right)$. Thus, the ratio between the lower and the upper bound for partial spreads tends to 1 if $v^{\prime}=v-k+d / 2$ tends to infinity. Since

$$
\begin{aligned}
& \left.\left\lfloor\frac{q^{v}-1}{q^{k}-1}\left\lfloor\frac{q^{v-1}-1}{q^{k-1}-1}\left\lfloor\ldots\left\lfloor\frac{q^{v-k+d / 2+1}-1}{q^{d / 2+1}-1}\left\lfloor\frac{q^{v-k+d / 2}-1}{q^{d / 2}-1}\right\rfloor\right\rfloor\right\rfloor\right\rfloor\right\rfloor\right\rfloor \\
\geq & \left(\frac{q^{v}-1}{q^{k}-1}\left(\frac{q^{v-1}-1}{q^{k-1}-1}\left(\ldots\left(\frac{q^{v-k+d / 2}-1}{q^{d / 2}-1}-1\right) \ldots\right)-1\right)-1\right)-1 \\
\geq & \frac{\left[\begin{array}{l}
v \\
k
\end{array}\right]_{q}}{\left[\begin{array}{c}
v-k+d / 2-1 \\
d / 2-1
\end{array}\right]_{q}}-(k-d / 2+1) \cdot \frac{\left[\begin{array}{c}
v-1 \\
k-1
\end{array}\right]_{q}}{\left[\begin{array}{c}
v-k+d / 2-1 \\
d / 2-1
\end{array}\right]_{q}} \\
\geq & \frac{\left[\begin{array}{l}
v \\
k
\end{array}\right]_{q}}{\left[\begin{array}{c}
v-k+d / 2-1 \\
d / 2-1
\end{array}\right]_{q}} \cdot\left(1-\frac{4(k-d / 2+1)}{q^{v-k}}\right)
\end{aligned}
$$

the ratio between Corollary 2 and the Anticode bound tends to 1 as $v$ tends to infinity.

Next, we consider the ratio between the lower bound from the first construction of Proposition 6 and the Anticode bound when $l$ tends to infinity. 
Proposition 9. For integers satisfying the conditions of Proposition $6, k \leq s$ and $d \leq 2 k$, we have

$$
\begin{aligned}
& \lim _{l \rightarrow \infty}\left(b(s)^{l} a\left(v_{0}\right)+a(s-d / 2+k)\left[\begin{array}{l}
l \\
1
\end{array}\right]_{b(s)}\right) / \frac{\left[\begin{array}{c}
v_{0}+l s \\
k
\end{array}\right]_{q}}{\left[\begin{array}{c}
v_{0}+l s-k+d / 2-1 \\
d / 2-1
\end{array}\right]_{q}} \\
& =\frac{a\left(v_{0}\right)+\frac{a(s-d / 2+k)}{q^{s(k-d / 2+1)}-1}}{q^{\left(v_{0}-k\right)(k-d / 2+1)}} \cdot \prod_{i=d / 2}^{k}\left(1-\frac{1}{q^{i}}\right)
\end{aligned}
$$

Proof. For $k \leq s$ and $k-d / 2+1 \geq 1$ we have $b(s) \neq 1$, so that

$$
\begin{array}{r}
b(s)^{l} a\left(v_{0}\right)+a\left(s^{\prime}\right)\left[\begin{array}{l}
l \\
1
\end{array}\right]_{b(s)}=q^{l s k^{\prime}} a\left(v_{0}\right)+a\left(s^{\prime}\right) \frac{q^{l s k^{\prime}}-1}{q^{s k^{\prime}}-1} \\
=q^{l s k^{\prime}}\left(a\left(v_{0}\right)+\frac{a\left(s^{\prime}\right)}{q^{s k^{\prime}}-1}\right)-\frac{a\left(s^{\prime}\right)}{q^{s k^{\prime}}-1},
\end{array}
$$

where we abbreviate $s^{\prime}=s-d / 2+k$ and $k^{\prime}=k-d / 2+1$. Thus,

$$
\lim _{l \rightarrow \infty}\left(b(s)^{l} a\left(v_{0}\right)+a\left(s^{\prime}\right)\left[\begin{array}{l}
l \\
1
\end{array}\right]_{b(s)}\right) / q^{l s k^{\prime}}=a\left(v_{0}\right)+\frac{a\left(s^{\prime}\right)}{q^{s k^{\prime}}-1} .
$$

Plugging in the definition of the $q$-binomial coefficients gives

$$
\frac{\left[\begin{array}{c}
v_{0}+l s \\
k
\end{array}\right]_{q}}{\left[\begin{array}{c}
v_{0}+l s-k+d / 2-1 \\
d / 2-1
\end{array}\right]_{q}}=\frac{\prod_{i=1}^{k} \frac{q^{v_{0}+l s-k+i}-1}{q^{i}-1}}{\prod_{i=1}^{d / 2-1} \frac{q^{v_{0}+l s-k+i}-1}{q^{i}-1}}=\prod_{i=d / 2}^{k} \frac{q^{v_{0}+l s-k+i}-1}{q^{i}-1}
$$

so that

$$
\lim _{l \rightarrow \infty} \frac{\left[\begin{array}{c}
v_{0}+l s \\
k
\end{array}\right]_{q}}{\left[\begin{array}{c}
v_{0}+l s-k+d / 2-1 \\
d / 2-1
\end{array}\right]_{q}} / q^{l s k^{\prime}}=\prod_{i=d / 2}^{k} \frac{q^{v_{0}-k+i}}{q^{i}-1}=q^{\left(v_{0}-k\right) k^{\prime}} \cdot \prod_{i=d / 2}^{k} \frac{1}{1-\frac{1}{q^{i}}} .
$$

Dividing both derived limits gives the proposed result.

For Example 1 with $d=4$ and $k=3$, we obtain a ratio of

$$
\left(1597245+\frac{A_{2}(7,4 ; 3)}{4095}\right) \cdot 21 / 2^{25} \in[0.99963386,0.99963388]
$$

for $v=13+6 l$ with $l \rightarrow \infty$ using $333 \leq A_{2}(7,4 ; 3) \leq 381$, i.e., the Anticode bound is almost met by the underlying improved linkage construction. 


\section{Codes better than the MRD bound}

For constant dimension codes that contain a lifted MRD code, Theorem 10 gives an upper bound which is tighter than the Johnson bound of Theorem 7. In [5] two infinite series of constructions have been given where the code sizes exceed the MRD bound of Theorem 10 for $q=2, d=4$, and $k=3$. Given the data available from 23 , we mention that, besides $d=4, k=3$, the only other case where the MRD bound was superseded is $A_{2}(8,4 ; 4) \geq 4801>4797$, see [10]. Next, we show that for $d=4$ and $k=3$ the MRD bound can be superseded for all field sizes $q$ if $v$ is large enough. For the limit of the achievable ratio we obtain:

Proposition 10. For $q \geq 3$ we have $\lim _{v \rightarrow \infty} \frac{A_{q}(v, 4 ; 3)}{q^{2 v-6}+\left[\begin{array}{c}v-3 \\ 2\end{array}\right]_{q}} \geq 1+\frac{1}{2 q^{3}}$.

Proof. For $q \geq 2$, [25, Theorem 4] gives

$$
A_{q}(7,4 ; 3) \geq q^{8}+q^{5}+q^{4}+q^{2}-q \geq q^{8}+q^{5}+q^{4} .
$$

With this, we conclude

$$
A_{q}\left(v_{0}, 4 ; 3\right) \geq A_{q}(7,4 ; 3) \cdot q^{2 v_{0}-14}+A_{q}\left(v_{0}-6,4 ; 3\right) \geq q^{2 v_{0}-10} \cdot\left(q^{4}+q+1\right)
$$

from Corollary 4 choosing $m=7$. Applying Proposition 6 with $s=3$ gives

$$
A_{q}\left(v_{0}+3 l, 4 ; 3\right) \geq q^{6 l} A_{q}\left(v_{0}, 4 ; 3\right)+\frac{q^{6 l}-1}{q^{6}-1} \geq q^{6 l} A_{q}\left(v_{0}, 4 ; 3\right)
$$

for $v_{0} \in\{12,13,14\}$, so that $A_{q}(v, 4 ; 3) \geq q^{2 v-10} \cdot\left(q^{4}+q+1\right)$ for all $v \geq 12$.

From Lemma 5 we conclude

$$
\lim _{v \rightarrow \infty} \frac{q^{2 v-6}+\left[\begin{array}{c}
v-3 \\
2
\end{array}\right]_{q}}{q^{2 v-10}}=q^{4}+(1 / q ; 1 / q)_{2}=\frac{q^{3}\left(q^{4}-q^{3}-q^{2}+q+1\right)}{(q-1)^{2}(q+1)} .
$$

Since

$$
\left(q^{4}+q+1\right) / \frac{q^{3}\left(q^{4}-q^{3}-q^{2}+q+1\right)}{(q-1)^{2}(q+1)}=1+\frac{1}{q^{3}}-\frac{q+1}{q^{2}\left(q^{4}-q^{3}-q^{2}+q+1\right)},
$$

the statement follows for $q \geq 3$.

For $q=2$ the estimations of the proof of Proposition 10 are too crude in order to obtain a factor larger than one. However, for the binary case better codes with moderate dimensions of the ambient space have been found by computer searches with the prescription of automorphisms as the main ingredient in order to reduce the computational complexity, see e.g. [31.

Proposition 11. For $v \geq 19$ we have $\frac{A_{2}(v, 4 ; 3)}{2^{2 v-6}+\left[\begin{array}{c}v-3 \\ 2\end{array}\right]_{2}} \geq 1.3056$. 
Proof. Applying Proposition 6 with $s=3$ and using $A_{2}(4,4 ; 3) \geq 0$ gives $A_{2}\left(v_{0}+3 l, 4 ; 3\right) \geq$ $A_{2}\left(v_{0}, 4 ; 3\right) \cdot 2^{6 l}$ for all $v_{0} \geq 6$ and $l \geq 0$, so that

$$
\frac{A_{2}\left(v_{0}+3 l, 4 ; 3\right)}{2^{2\left(v_{0}+3 l\right)-6}+\left[\begin{array}{c}
\left(v_{0}+3 l\right)-3 \\
2
\end{array}\right]_{2}} \geq \frac{A_{2}\left(v_{0}, 4 ; 3\right)}{\frac{7}{3} \cdot 2^{2 v_{0}-7}} .
$$

Using $A_{2}(7,4 ; 3) \geq 333$ [23], $A_{2}(8,4 ; 3) \geq 1326$ [10], $A_{2}(9,4 ; 3) \geq 5986$ [10], and $A_{2}(13,4 ; 3)=1597245$ [9] we apply Corollary 4 with $m=13$ to obtain lower bounds for $A_{2}\left(v_{0}, 4 ; 3\right)$ with $19 \leq v_{0} \leq 21$. For these values of $v_{0}$ the minimum of the right hand side of Inequality (9) is attained at $v_{0}=20$ with value 1.3056442377 .

Note that the application of Proposition 6 was used in a rather crude estimation in the proof of Proposition 11. Actually, we do not use the codewords generated by the codewords of cdc $C_{2}$ in Theorem 18, so that we might have applied [38, Theorem 37] directly for this part of the proof - similarly for Proposition 10, which then allows to consider just one instead of $s=3$ starters. In the latter part of the proof of Proposition 11 the use of Corollary 4 is essential in order to obtain large codes for medium sized dimensions of the ambient space from $A_{2}(13,4 ; 3)=1597245$ and relatively good lower bounds for small dimensions. This is a relative typical behavior of Corollary 4 and Proposition 6, i.e., the first few applications yield a significant improvement which quickly bottoms out - in a certain sense. As column bklb of Table 3 suggests, we may slightly improve upon the value stated in Proposition 11 by some fine-tuning effecting the omitted less significant digits.

\begin{tabular}{l|lll|ll|l}
$v$ & bklb & mrdb & bkub & lold & lnew & ea \\
\hline 6 & 77 & 71 & 77 & 65 & 65 & \\
7 & 333 & 291 & 381 & 257 & 265 & 301 \\
8 & 1326 & 1179 & 1493 & 1033 & 1101 & 1117 \\
9 & 5986 & 4747 & 6205 & 4929 & 4929 & 4852 \\
10 & 23870 & 19051 & 24698 & 21313 & 21313 & 18924 \\
11 & 97526 & 76331 & 99718 & 85249 & 85257 & 79306 \\
12 & 385515 & 305579 & 398385 & 383105 & 383105 & 309667 \\
13 & 1597245 & 1222827 & 1597245 & 1532417 & 1532425 & 1287958 \\
14 & 6241665 & 4892331 & 6387029 & 6241665 & 6241665 & 4970117 \\
15 & 24966665 & 19571371 & 25562941 & 24966657 & 24966665 & 20560924 \\
16 & 102223681 & 78289579 & 102243962 & 102223681 & 102223681 & 79608330 \\
17 & 408894729 & 313166507 & 409035142 & 408894721 & 408894729 & \\
18 & 1635578957 & 1252682411 & 1636109361 & 1635578889 & 1635578957 & \\
19 & 6542315853 & 5010762411 & 6544674621 & 6542315597 & 6542315853 & 5200895489
\end{tabular}

Table 1: Lower and upper bounds for $A_{2}(v, 4 ; 3)$. 
In Tables 1, 2, and 3 we compare the sizes of different constructions with the LMRD and the best known upper bound. Here bklb and bkub stand for best known lower and upper bound respectively. The values of Theorem 10 are given in column mrdb. Applying Theorem 17 and Theorem 18 to the best known codes give the columns lold and lnew, respectively. The results obtained in [5] are stated in column ea. The achieved ratio between the mentioned constructions and the MRD bound can be found in Table 3 , Since differences partially are beyond the given accuracy, we give absolute numbers in Table 1 . Note that the values in column bklb of Table 3 show that Proposition 11 is also valid for $v \geq 16$, while we have a smaller ratio for $v<16$. The relative advantage over lifted MRD codes is displayed in Table 2 .

\begin{tabular}{l|lll|ll|l}
$v$ & bklb & mrdb & bkub & lold & lnew & ea \\
\hline 6 & 1.203125 & 1.109375 & 1.203125 & 1.015625 & 1.015625 & \\
7 & 1.300781 & 1.136719 & 1.488281 & 1.003906 & 1.035156 & 1.175781 \\
8 & 1.294922 & 1.151367 & 1.458008 & 1.008789 & 1.075195 & 1.090820 \\
9 & 1.461426 & 1.158936 & 1.514893 & 1.203369 & 1.203369 & 1.184570 \\
10 & 1.456909 & 1.162781 & 1.507446 & 1.300842 & 1.300842 & 1.155029 \\
11 & 1.488129 & 1.164719 & 1.521576 & 1.300797 & 1.300919 & 1.210114 \\
12 & 1.470623 & 1.165691 & 1.519718 & 1.461430 & 1.461430 & 1.181286 \\
13 & 1.523252 & 1.166179 & 1.523252 & 1.461427 & 1.461434 & 1.228292 \\
14 & 1.488129 & 1.166423 & 1.522786 & 1.488129 & 1.488129 & 1.184968 \\
15 & 1.488129 & 1.166545 & 1.52367 & 1.488129 & 1.488129 & 1.225527 \\
16 & 1.523252 & 1.166606 & 1.523554 & 1.523252 & 1.523252 & 1.186257 \\
17 & 1.523252 & 1.166636 & 1.523775 & 1.523252 & 1.523252 & \\
18 & 1.523252 & 1.166651 & 1.523746 & 1.523252 & 1.523252 & \\
19 & 1.523252 & 1.166659 & 1.523801 & 1.523252 & 1.523252 & 1.210928
\end{tabular}

Table 2: Lower and upper bounds for $A_{2}(v, 4 ; 3)$ divided by the size of a corresponding lifted MRD code.

To conclude this section, we remark that an application of Corollary 4 with $2 k \leq m \leq$ $v-k$ using a lifted MRD in the cdc $C_{1}$ cannot generate a code that exceeds the MRD bound of Theorem 10 .

Lemma 6. Using the notation of Theorem 18, let $k \leq \min \left\{v_{1}-k, v_{2}-k+d / 2\right\}, C_{r}$ a linear $M R D$ code, $d_{r}=d_{1} / 2$, and $C_{1}$ contains a lifted $M R D$ code (in $\left[\begin{array}{c}\mathbb{F}_{q}^{v_{1}} \\ k\end{array}\right]$ ). Then, the codes constructed in Theorem 18 contain a lifted $M R D$ code $\left(\right.$ in $\left.\left[\begin{array}{c}\mathbb{F}_{q}^{v_{1}+v_{2}-k+d / 2} \\ k\end{array}\right]\right)$.

Proof. Let $\left\{\tau^{-1}\left(I_{k \times k} \mid M\right): M \in R\right\} \subseteq C_{1}$ be the lifted MRD code in $C_{1}$. Since $R$ is a $\left(k \times\left(v_{1}-k\right), d_{1} / 2\right)_{q}$ MRD code, we have $\# R=q^{\left(v_{1}-k\right)\left(k-d_{1} / 2+1\right)}$. The first set of the construction contains

$$
\left\{\tau^{-1}\left(I_{k \times k}|M| A\right): M \in R, A \in C_{r}\right\}
$$


in which $\left\{(M \mid A): M \in R, A \in C_{r}\right\}$ forms a $\left(k \times\left(v_{1}+v_{2}-2 k+d / 2\right), N, d_{r}\right)_{q}$ rank metric code of size $N=q^{\left(v_{1}+v_{2}-2 k+d / 2\right)\left(k-d_{r}+1\right)}$, hence it is a maximum rank metric code.

\begin{tabular}{l|lll|ll|l}
$v$ & bklb & mrdb & bkub & lold & lnew & ea \\
\hline 6 & 1.084507 & 1.0 & 1.084507 & 0.915493 & 0.915493 & \\
7 & 1.144330 & 1.0 & 1.309278 & 0.883162 & 0.910653 & 1.034364 \\
8 & 1.124682 & 1.0 & 1.266327 & 0.876166 & 0.933842 & 0.947413 \\
9 & 1.261007 & 1.0 & 1.307141 & 1.038340 & 1.038340 & 1.022119 \\
10 & 1.252953 & 1.0 & 1.296415 & 1.118734 & 1.118734 & 0.993334 \\
11 & 1.277672 & 1.0 & 1.306389 & 1.116833 & 1.116938 & 1.038975 \\
12 & 1.261589 & 1.0 & 1.303705 & 1.253702 & 1.253702 & 1.013378 \\
13 & 1.306190 & 1.0 & 1.306190 & 1.253176 & 1.253182 & 1.053263 \\
14 & 1.275806 & 1.0 & 1.305519 & 1.275806 & 1.275806 & 1.015900 \\
15 & 1.275673 & 1.0 & 1.306140 & 1.275672 & 1.275673 & 1.050561 \\
16 & 1.305712 & 1.0 & 1.305972 & 1.305712 & 1.305712 & 1.016845 \\
17 & 1.305678 & 1.0 & 1.306127 & 1.305678 & 1.305678 & \\
18 & 1.305661 & 1.0 & 1.306085 & 1.305661 & 1.305661 & \\
19 & 1.305653 & 1.0 & 1.306124 & 1.305653 & 1.305653 & 1.037945
\end{tabular}

Table 3: Lower and upper bounds for $A_{2}(v, 4 ; 3)$ divided by the corresponding MRD bound.

\section{Conclusion}

In this paper we have considered the maximal sizes of constant dimension codes. With respect to constructive lower bounds we have improved the so-called linkage construction, which then yields the best known codes for many parameters, see Footnote 2. With respect to upper bounds there is a rather clear picture. The explicit Corollary 2, which refers back to bounds for partial spreads, is the best known parametric bound in the case of $d \neq 2 \min \{k, v-k\}$, while Theorem 8 or the linear programming method may possibly yield improvements. Since Theorem 8 implies the Johnson bound and so Corollary 2 , it would be worthwhile to study whether it can be strictly sharper than Theorem 7 for $d \neq 2 \min \{k, v-k\}$ at all. Compared to Corollary 2, the only two known improvements are given for the specific parameters from Theorem 9 and Proposition 5. In the case of partial spreads we have reported the current state-of-the-art mentioning that further improvements are far from being unlikely.

In general we have shown that the ratio between the best-known lower and upper bound is strictly larger than 0.577576 for all parameters. The bottleneck is formed by the parameters $q=2, d=4$, and $k=\lfloor v / 2\rfloor$, where no known method can properly improve that factor, see Footnote 2 for the linkage construction. For $d=4, k=3$ and general 
field sizes $q$ we have applied the improved linkage construction in order to show that $A_{q}(v, d ; k)$ is by a factor, depending on $q$, larger than the MRD bound for sufficiently large dimensions $v$.

\section{Acknowledgement}

The authors would like to thank Harout Aydinian for providing an enlarged proof of Theorem 8, Natalia Silberstein for explaining the restriction $3 k \leq v$ in [38, Corollary 39], Heide Gluesing-Luerssen for clarifying the independent origin of the linkage construction, and Alfred Wassermann for discussions about the asymptotic results of Frankl and Rödl.

\section{References}

[1] E. Agrell, A. Vardy, and K. Zeger. Upper bounds for constant-weight codes. IEEE Transactions on Information Theory, 46(7):2373-2395, 2000.

[2] R. Ahlswede and H. Aydinian. On error control codes for random network coding. In Network Coding, Theory, and Applications, 2009. NetCod'09. Workshop on, pages 68-73. IEEE, 2009.

[3] R. Ahlswede, H. K. Aydinian, and L. H. Khachatrian. On perfect codes and related concepts. Designs, Codes and Cryptography, 22(3):221-237, 2001.

[4] R. Ahlswede, N. Cai, S.-Y. R. Li, and R. W. Yeung. Network information flow. IEEE Transactions on Information Theory, 46(4):1204-1216, 2000.

[5] J. Ai, T. Honold, and H. Liu. The expurgation-augmentation method for constructing good plane subspace codes. arXiv preprint 1601.01502, 2016.

[6] C. Bachoc, A. Passuello, and F. Vallentin. Bounds for projective codes from semidefinite programming. Advances in Mathematics of Communications, 7(2):127$145,2013$.

[7] A. Beutelspacher. Partial spreads in finite projective spaces and partial designs. Mathematische Zeitschrift, 145(3):211-229, 1975.

[8] S. R. Blackburn and T. Etzion. The asymptotic behavior of grassmannian codes. IEEE Transactions on Information Theory, 58(10):6605-6609, 2012.

[9] M. Braun, T. Etzion, P. R. J. Östergård, A. Vardy, and A. Wassermann. Existence of $q$-analogs of steiner systems. Forum of Mathematics, Pi, 4, 2016.

[10] M. Braun, P. R. J. Östergård, and A. Wassermann. New lower bounds for binary constant-dimension subspace codes. Experimental Mathematics, 0(0):1-5, 0.

[11] P. Delsarte. An algebraic approach to the association schemes of coding theory. PhD thesis, Philips Research Laboratories, 1973. 
[12] P. Delsarte. Hahn polynomials, discrete harmonics, and t-designs. SIAM Journal on Applied Mathematics, 34(1):157-166, 1978.

[13] D. Drake and J. Freeman. Partial $t$-spreads and group constructible $(s, r, \mu)$-nets. Journal of Geometry, 13(2):210-216, 1979.

[14] S. El-Zanati, H. Jordon, G. Seelinger, P. Sissokho, and L. Spence. The maximum size of a partial 3-spread in a finite vector space over $G F(2)$. Designs, Codes and Cryptography, 54(2):101-107, 2010.

[15] T. Etzion and N. Silberstein. Error-correcting codes in projective spaces via rankmetric codes and Ferrers diagrams. IEEE Transactions on Information Theory, 55(7):2909-2919, 2009.

[16] T. Etzion and N. Silberstein. Codes and designs related to lifted MRD codes. IEEE Transactions on Information Theory, 59(2):1004-1017, 2013.

[17] T. Etzion and A. Vardy. Error-correcting codes in projective space. IEEE Transactions on Information Theory, 57(2):1165-1173, 2011.

[18] P. Frankl and V. Rödl. Near perfect coverings in graphs and hypergraphs. European Journal of Combinatorics, 6(4):317-326, 1985.

[19] P. Frankl and R. M. Wilson. The Erdős-Ko-Rado theorem for vector spaces. Journal of Combinatorial Theory, Series A, 43(2):228-236, 1986.

[20] E. Gabidulin. Theory of codes with maximum rank distance. Problemy Peredachi Informatsii, 21(1):3-16, 1985.

[21] H. Gluesing-Luerssen, K. Morrison, and C. Troha. Cyclic orbit codes and stabilizer subfields. Advances in Mathematics of Communications, 9(2):177-197, 2015.

[22] H. Gluesing-Luerssen and C. Troha. Construction of subspace codes through linkage. Advances in Mathematics of Communications, 10(3):525-540, 2016.

[23] D. Heinlein, M. Kiermaier, S. Kurz, and A. Wassermann. Tables of subspace codes. arXiv preprint 1601.02864, 2016.

[24] D. Heinlein and S. Kurz. A new upper bound for subspace codes. arXiv preprint 1703.08712, 2017.

[25] T. Honold and M. Kiermaier. On putative $q$-analogues of the Fano plane and related combinatorial structures. In Dynamical systems, number theory and applications, pages 141-175. World Sci. Publ., Hackensack, NJ, 2016.

[26] T. Honold, M. Kiermaier, and S. Kurz. Optimal binary subspace codes of length 6 , constant dimension 3 and minimum subspace distance 4. In Topics in finite fields, volume 632 of Contemp. Math., pages 157-176. Amer. Math. Soc., Providence, RI, 2015. 
[27] T. Honold, M. Kiermaier, and S. Kurz. Partial spreads and vector space partitions. arXiv preprint 1611.06328, 2016.

[28] S. Johnson. A new upper bound for error-correcting codes. IRE Transactions on Information Theory, 8(3):203-207, 1962.

[29] A. Khaleghi, D. Silva, and F. Kschischang. Subspace codes. In IMA International Conference on Cryptography and Coding, pages 1-21. Springer, 2009.

[30] M. Kiermaier, S. Kurz, and A. Wassermann. The order of the automorphism group of a binary $q$-analog of the fano plane is at most two. Designs, Codes and Cryptography, to appear.

[31] A. Kohnert and S. Kurz. Construction of large constant dimension codes with a prescribed minimum distance. In Mathematical methods in computer science, volume 5393 of Lecture Notes in Computer Science, pages 31-42. Springer, Berlin, 2008.

[32] R. Kötter and F. R. Kschischang. Coding for errors and erasures in random network coding. IEEE Transactions on Information Theory, 54(8):3579-3591, 2008.

[33] S. Kurz. Packing vector spaces into vector spaces. The Australasian Journal of Combinatorics, 68(1):122-130, 2017.

[34] S. Kurz. Improved upper bounds for partial spreads. Designs, Codes and Cryptography, to appear.

[35] F. J. MacWilliams and N. J. A. Sloane. The theory of error-correcting codes. II. North-Holland Publishing Co., Amsterdam-New York-Oxford, 1977. North-Holland Mathematical Library, Vol. 16.

[36] E. Năstase and P. Sissokho. The maximum size of a partial spread in a finite projective space. arXiv preprint 1605.04824, 2016.

[37] B. Segre. Teoria di galois, fibrazioni proiettive e geometrie non desarguesiane. Annali di Matematica Pura ed Applicata, 64(1):1-76, 1964.

[38] N. Silberstein and A.-L. Trautmann. Subspace codes based on graph matchings, ferrers diagrams, and pending blocks. IEEE Transactions on Information Theory, 61(7):3937-3953, 2015.

[39] D. Silva, F. Kschischang, and R. Kötter. A rank-metric approach to error control in random network coding. IEEE Transactions on Information Theory, 54(9):3951-3967, 2008.

[40] D. Silva and F. R. Kschischang. On metrics for error correction in network coding. IEEE Transactions on Information Theory, 55(12):5479-5490, 2009.

[41] V. D. Tonchev. Codes and designs. Handbook of coding theory, 2:1229-1267, 1998. 
[42] H. Wang, C. Xing, and R. Safavi-Naini. Linear authentication codes: bounds and constructions. IEEE Transactions on Information Theory, 49(4):866-872, 2003.

[43] S.-T. Xia and F.-W. Fu. Johnson type bounds on constant dimension codes. Designs, Codes and Cryptography, 50(2):163-172, 2009. 